The Jeffery-Hamel similarity solution and its relation to flow in a diverging channel

Haines, P.E. and Hewitt, R.E. and Hazel, A.L.

2011

MIMS EPrint: 2013.61

Manchester Institute for Mathematical Sciences

School of Mathematics

The University of Manchester

\footnotetext{
Reports available from: http://eprints.maths.manchester.ac.uk/

And by contacting: The MIMS Secretary

School of Mathematics

The University of Manchester

Manchester, M13 9PL, UK
} 


\title{
The Jeffery-Hamel similarity solution and its relation to flow in a diverging channel
}

\author{
P. E. Haines $\dagger \ddagger$, R. E. Hewitt and A. L. Hazel \\ School of Mathematics, University of Manchester, Manchester M13 9PL, UK
}

(Received 12 December 2010; revised 11 July 2011; accepted 26 August 2011)

\begin{abstract}
We explore the relevance of the idealized Jeffery-Hamel similarity solution to the practical problem of flow in a diverging channel of finite (but large) streamwise extent. Numerical results are presented for the two-dimensional flow in a wedge of separation angle $2 \alpha$, bounded by circular arcs at the inlet/outlet and for a net radial outflow of fluid. In particular, we show that in a finite domain there is a sequence of nested neutral curves in the $(R e, \alpha)$ plane, each corresponding to a midplane symmetry-breaking (pitchfork) bifurcation, where $R e$ is a Reynolds number based on the radial mass flux. For small wedge angles we demonstrate that the first pitchfork bifurcation in the finite domain occurs at a critical Reynolds number that is in agreement with the only pitchfork bifurcation in the infinite-domain similarity solution, but that the criticality of the bifurcation differs (in general). We explain this apparent contradiction by demonstrating that, for $\alpha \ll 1$, superposition of two (infinite-domain) eigenmodes can be used to construct a leading-order finite-domain eigenmode. These constructed modes accurately predict the multiple symmetry-breaking bifurcations of the finite-domain flow without recourse to computation of the full field equations. Our computational results also indicate that temporally stable, isolated, steady solutions may exist. These states are finite-domain analogues of the steady waves recently presented by Kerswell, Tutty, \& Drazin (J. Fluid Mech., vol. 501, 2004, pp. 231-250) for an infinite domain. Moreover, we demonstrate that there is non-uniqueness of stable solutions in certain parameter regimes. Our numerical results tie together, in a consistent framework, the disparate results in the existing literature.
\end{abstract}

Key words: bifurcation, general fluid mechanics

\section{Introduction}

The Jeffery-Hamel similarity solution is an exact solution to the Navier-Stokes equations that describes the two-dimensional flow of an incompressible viscous

$\dagger$ Email address for correspondence: philip.haines@adelaide.edu.au

$\$$ Present address: School of Mathematical Sciences, University of Adelaide, Adelaide, SA 5005, Australia. 


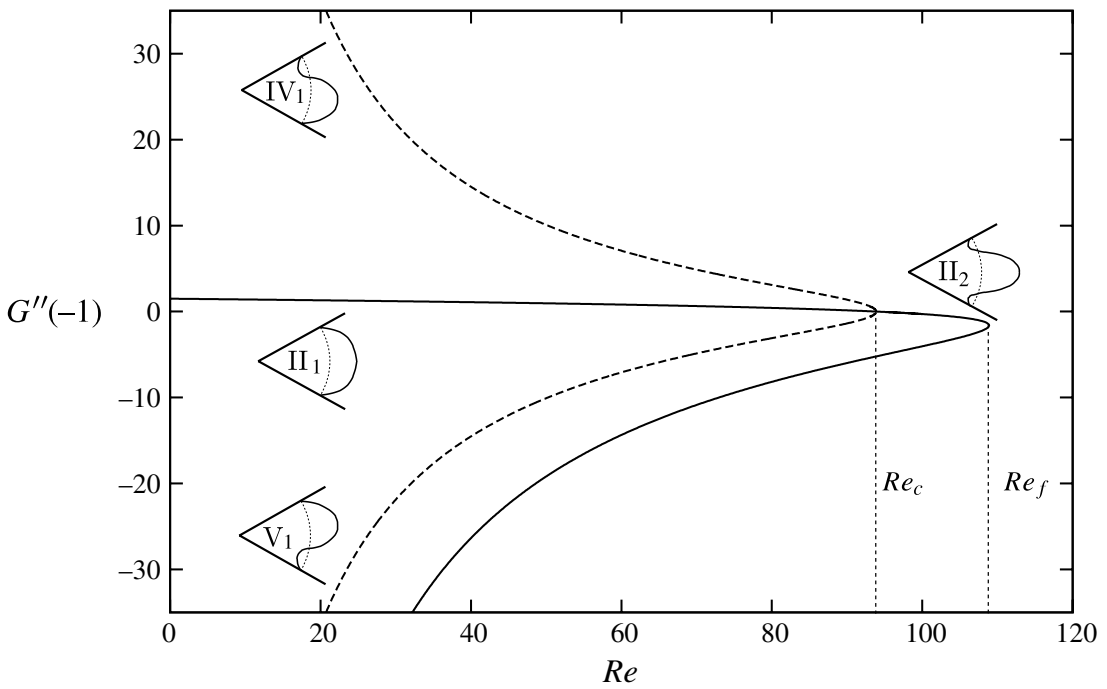

FIGURE 1. Jeffery-Hamel flows, solutions of (1.2), for $\alpha=0.1$; the metric we choose here is $G^{\prime \prime}(-1)$, which is related to the shear stress distribution at the 'lower' wall $\phi=-1$. Here $R e_{c}$ denotes the location of a subcritical symmetry-breaking bifurcation and $R e_{f}$ refers to the location of the turning point in the symmetric solution branch. The inset figures are sketches of the radial flow profile associated with each solution branch. The labels refer to the classification of the different flows given by Fraenkel (1962).

fluid between two semi-infinite diverging planes. Jeffery-Hamel flows were first documented almost a century ago (Jeffery 1915; Hamel 1916), since which time the governing ordinary differential equation has been studied extensively.

In terms of polar coordinates, $(\hat{r}, \theta)$, centred at the apex of an infinite wedge of separation angle $2 \alpha$, the Jeffery-Hamel form of the streamfunction $\psi$ is

$$
\psi(\hat{r}, \theta)=Q G(\phi) .
$$

Here $Q$ is a constant radial 'volume' flux and, following Fraenkel (1962), $\phi=\theta / \alpha \in$ $[-1,1]$ is a rescaled angle. The function $G(\phi)$ satisfies

$$
G^{(i v)}+2 \alpha \operatorname{Re} G^{\prime} G^{\prime \prime}+4 \alpha^{2} G^{\prime \prime}=0,
$$

where primes indicate differentiation with respect to $\phi$, and $R e=Q / v$ is a Reynolds number. The appropriate boundary conditions are

$$
G( \pm 1)= \pm \frac{1}{2} \quad G^{\prime}( \pm 1)=0,
$$

describing no slip and no penetration at the wedge walls.

Although the system (1.2) is known to have an infinite number of solutions for any value of the Reynolds number (Fraenkel 1962), we are most concerned with those solutions that can be reached by continuous variation from the Stokes solution; as shown in figure 1 for the representative case of $\alpha=0.1$.

These self-similar flows have been fully classified (Rosenhead 1940; Fraenkel 1962), as has the bifurcation structure that connects them (Banks, Drazin \& Zaturska 1988; Goldshtik, Hussain \& Shtern 1991). The most basic Jeffery-Hamel solution ( $\mathrm{II}_{1}$ ) for a net mass flux away from the wedge apex consists of a midplane symmetric outflow across the entire channel. For a fixed channel angle, this pure outflow solution can 
only be found below a critical Reynolds number, $\operatorname{Re}_{c}(\alpha)$. Above this critical value the solution develops a region of reverse flow at both of the wedge walls $\left(\mathrm{II}_{2}\right)$. In addition, there is also a subcritical pitchfork bifurcation at $R e_{c}$ and two solutions $\left(\operatorname{IV}_{1}\right.$ and $\left.\mathrm{V}_{1}\right)$ that break the symmetry about the midplane of the channel exist for $\operatorname{Re}<e_{c}(\alpha)$. A turning point is present at a larger Reynolds number $\operatorname{Re}_{f}(\alpha)$ but will play a limited role herein. In the $(R e, \alpha)$ plane, the locus of points $\left(\operatorname{Re}_{c}(\alpha), \alpha\right)$ form a boundary that (following Fraenkel) we denote by $\mathscr{B}_{2}$. As $\alpha \rightarrow 0$ it is known that $\operatorname{Re}_{c}(\alpha) \sim 3 \pi / \alpha$ (Fraenkel 1962; Akulenko \& Kumakshev 2008).

The Jeffery-Hamel solutions describe a flow in an idealized, infinite, domain rather than the practically relevant case of a system of finite streamwise extent. A plausible, often implicit, assumption is that for 'sufficiently long' domains the results of the ordinary-differential system (1.2) are relevant to the problem in a finite domain. Various studies, both numerical and asymptotic, have attempted to relate Jeffery-Hamel solutions to the study of flow structure and stability in expanding channels of finite length. Banks et al. (1988) addressed the spatial stability of symmetric Jeffery-Hamel flows by allowing for linearized perturbations of the form $r^{\lambda} g(\phi)$, as first suggested by Dean (1934). Their work demonstrated that a loss of stability to these 'Dean modes' occurs at the pitchfork bifurcation boundary $\mathscr{B}_{2}$. Banks et al. (1988) computed the corresponding eigenmodes, but only for small or zero Reynolds number. The work of Goldshtik et al. (1991) subsequently presented limited results at Reynolds numbers for which the flow is fully nonlinear. Later, Dennis et al. (1997) performed a numerical study of the steady flow in a wedge for ratios of outlet to inlet radii between 7 and 25 . They examined the effect of imposing a small perturbation across either the inlet or the outlet in order to explore the relevance of the Dean modes in a finite domain. Their attempt to recover these modes resulted in limited success. They observed the loss of stability of the symmetric base flow to antisymmetric perturbations but were unable to provide a critical Reynolds number, specifying only that for $\alpha=\pi / 18$ the flow was stable to such perturbations for $R e=50$ and unstable for $R e=100$.

Both Sobey \& Drazin (1986) and Banks et al. (1988) identified the boundary $\mathscr{B}_{2}$ as the point at which the Jeffery-Hamel solution should cease to provide a good description of the flow in an expanding channel. Several studies have investigated the flow as the Reynolds number is increased beyond $\mathscr{B}_{2}$. Sobey \& Drazin (1986) performed numerical computations and experiments for a finite two-dimensional (smoothly) expanding channel, but only for an expansion ratio (the outlet width divided by the inlet width) of less than or equal to three. In these short domains a supercritical pitchfork bifurcation was observed to exist both numerically and experimentally. Although some critical values of the Reynolds number are given, it has since been suggested that their numerical results, and in particular the critical values obtained, may be inaccurate due to their use of an upwind differencing scheme (Sobey \& Mullin 1993). In the later numerical study of Dennis et al. (1997) symmetry-broken solutions were located at Reynolds numbers significantly greater than $R e_{c}$, reinforcing the opinion emerging from the literature that the bifurcation is a supercritical pitchfork for finite domains. However, their work did not compute the bifurcation directly and no critical parameter values were provided.

Hamadiche, Scott \& Jeandel (1994) presented the results of unsteady calculations in a wedge of finite length, finding a neutral curve for temporal stability in good agreement with $\mathscr{B}_{2}$ for small $\alpha$. However, they observed time-periodic nonlinear states for Reynolds numbers greater than the critical value perhaps indicating that they 
believed the loss of stability to arise via a Hopf bifurcation, although this is not stated explicitly.

An important advance was made by Tutty (1996) in an investigation of finitedomain flows at Reynolds numbers close to $R e_{c}$. Tutty observed the existence of a steady solution, which at large distances from the inlet appeared to be spatially periodic (on a logarithmic scale) in the radial coordinate. For values of the Reynolds number greater than $R e_{c}$ this spatially periodic solution was the one observed in his numerical work, suggesting some form of instability of the symmetric Jeffery-Hamel solution. For Reynolds numbers less than the critical value these 'steady waves' were only observed when the inlet geometry incorporated a sudden step expansion. Tutty (1996) interpreted the existence of the wave for Reynolds numbers less than $R e_{c}$ as support for the claim that the flow becomes unstable subcritically. Kerswell, Tutty \& Drazin (2004) furthered the work of Tutty by enforcing the spatial periodicity in the computational formulation. They found that the steady, spatially periodic solutions first observed by Tutty arise from a heteroclinic bifurcation across the arms of the subcritical Jeffery-Hamel pitchfork. They conjectured that such solutions may be realized in an experiment since they appear to be spatially stable for parameter values at which the symmetric Jeffery-Hamel solution is not.

In the extensive literature discussed above there are a number of clear discrepancies. In particular, there are contradictions regarding the bifurcation sequence obtained as the flow rate is increased, as highlighted by the supercritical pitchfork inferred by Sobey \& Drazin (1986) and Dennis et al. (1997), the supercritical Hopf bifurcation observed by Hamadiche et al. (1994) and the symmetry-broken 'spatial wave' found by Tutty (1996) and Kerswell et al. (2004). Whilst the available experimental evidence (Sobey \& Drazin 1986; Putkaradze \& Vorobieff 2006) qualitatively supports a supercritical pitchfork bifurcation, neither Sobey \& Drazin nor Dennis et al. gave values for the critical Reynolds number of this bifurcation. There is currently no study that fully describes the loss of stability of the symmetric outflow in an expanding channel or gives a comprehensive view of the solution structure. We address this by performing two-dimensional computations in a wedge-shaped domain bounded by two circular arcs. Our aim is to determine accurately the complete bifurcation structure and provide well-resolved values for the associated critical Reynolds numbers over a range of channel angles and domain sizes $(\S 3)$. In $\S 4$ we provide details of isolated solution branches that remain disconnected from those described in $\S 3$. We discuss the relevance of these numerical results to experimental configurations in $\S 5$.

After presenting the details of our numerical investigation, we return to the similarity solution $(\S 6)$ and discuss its relevance to the finite-domain flow. In particular, we show that, for low wedge angles, the bifurcation in a finite domain is dominated by the superposition of just two complex (radially oscillatory) Dean eigenmodes. Consequently, the loss of symmetry of the radial outflow solution in a finite domain can be predicted from the ordinary differential equation for the similarity solution without recourse to the full two-dimensional Navier-Stokes equations.

\section{Formulation of the finite-domain problem}

To aid comparison with Jeffery-Hamel flows we choose the geometry to be a finite section of a wedge bounded by an inlet and an outlet of constant radius as depicted in figure 2. The numerical problem is formulated in terms of polar coordinates $(\hat{r}, \theta)$ centred on the (virtual) apex of the wedge with corresponding velocities $(\hat{u}, \hat{v})$ and pressure $\hat{p}$. 


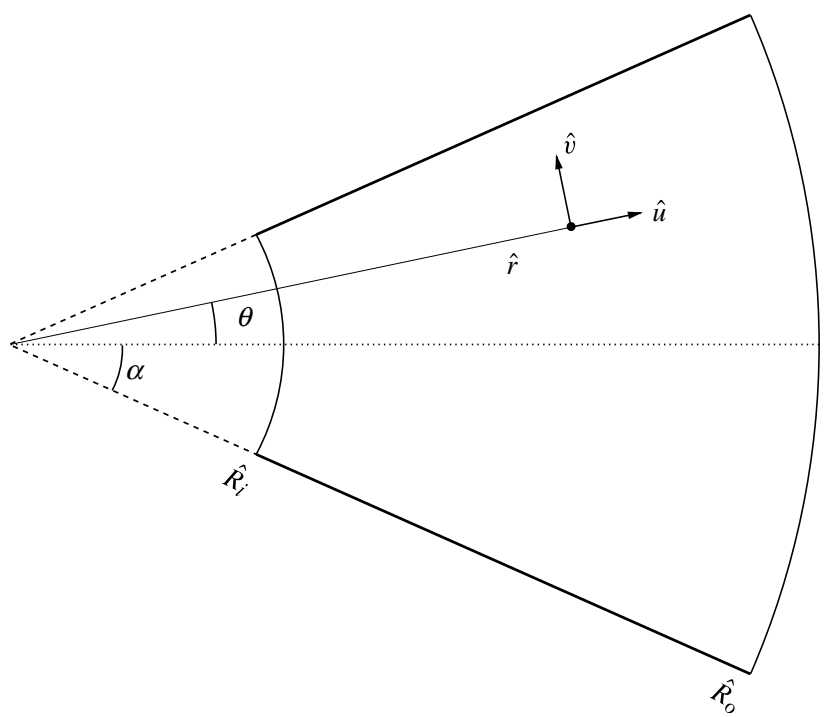

FIGURE 2. The two-dimensional geometry. Two parallel walls separated by a fixed angle $2 \alpha$ for which $\hat{R}_{i} \leqslant \hat{r} \leqslant \hat{R}_{o}$, where $\hat{r}$ is the radial coordinate of a set of polar coordinates centred at the (virtual) intersection of the two walls. The circular arcs at $\hat{R}_{i}$ and $\hat{R}_{o}$ are referred to as the inlet and outlet boundaries respectively. After non-dimensionalization, the size of the domain will be parameterized by the radius ratio $\eta=\hat{R}_{o} / \hat{R}_{i}$.

The finite-domain problem is non-dimensionalized in terms of the inlet 'volume' flux $Q$ and the outlet radius $\hat{R}_{o}$. The relevant velocity scale is then $U=Q / \hat{R}_{o}$ so the dimensionless radial coordinate, velocity and pressure are

$$
r=\frac{\hat{r}}{\hat{R}_{o}}, \quad \boldsymbol{u}=\frac{\hat{\boldsymbol{u}}}{U}, \quad p=\frac{\hat{R}_{o}}{U \rho \nu} \hat{p},
$$

where a hat indicates the corresponding dimensional variable. We also make use of a rescaled angle $\phi=\theta / \alpha$, such that $\boldsymbol{u}=\boldsymbol{u}(r, \phi)$ and $p=p(r, \phi)$.

The parameter space for the computational problem is spanned by

$$
\alpha, \quad R e=\frac{Q}{v}, \quad \eta=\frac{\hat{R}_{o}}{\hat{R}_{i}},
$$

which are the wedge half-angle, a Reynolds number and the outlet/inlet radius ratio. We solve the resulting dimensionless form of the Navier-Stokes equations (in polar form) using a finite-element method. The computational mesh is rectangular in the coordinates $r$ and $\phi$ and comprises (initially) $N_{r}$ elements in the radial direction and $N_{\phi}$ elements in the azimuthal direction. Where necessary the meshes undergo adaptive refinement as part of the solution procedure, using the $Z^{2}$ error estimator developed by Zienkiewicz \& Zhu (1992) with a flux based upon the rate of strain tensor. Initial mesh nodes are spaced logarithmically in the radial direction in order to minimize the error associated with resolving the inverse radius scaling of the Jeffery-Hamel similarity solution, however the initial azimuthal spacing is generally uniform. To better resolve rapid adjustments caused by the outlet boundary conditions, an outlet region with smaller radial spacing between the nodes was incorporated into the initial 
mesh structure. A typical mesh resolution was of the order $N_{r}=60, N_{\phi}=30$ prior to any adaptation.

The finite-element library oomph-lib (Heil \& Hazel 2006) was used to assemble and solve the system of algebraic equations associated with a weak form of the Navier-Stokes equations in plane polar coordinates on the finite domain, see Haines (2010) for details.

\subsection{Boundary conditions for the finite-domain numerical results}

In our finite-element formulation, the implied, so-called natural, boundary condition is for the flow to be 'pseudo-traction free' (PTF); and at the inlet/outlet of the finite domain, this gives

$$
-p+\frac{\partial u}{\partial r}=0, \quad \frac{\partial v}{\partial r}=0 .
$$

For the bulk of our finite-domain results in the following we impose the PTF condition (2.3) at the outlet (as is common for open channel flow computations; see Gresho \& Sani 1998, pp. 475-482) together with a Dirichlet constraint at the inlet. In any given experimental configuration it is unlikely that much control would be available regarding the exact nature of the profile at the inlet to the diverging channel. We therefore choose to introduce the following ad-hoc quadratic inlet condition:

$$
u=\frac{3}{4 \alpha r}\left(1-\phi^{2}\right), \quad v=0 \quad \text { on } r=\eta^{-1},
$$

on the grounds that this is a simple profile that lacks any special features. The factor of $\frac{3}{4}$ in (2.4) is required to impose a volume flux of unity. In the subsequent text we shall refer to this inlet condition as 'quadratic'.

We have repeated the results presented herein for several other plausible inlet/outlet conditions. The behaviour that we describe in the following section is generic in that it is relatively insensitive to the details of the boundary conditions, unless the conditions are specifically (artificially) chosen to reproduce the Jeffery-Hamel similarity solution. Moreover, the differences induced by changes in generic boundary conditions become less significant for smaller channel angles. We return to a discussion of variations in the boundary conditions in $\S 5$ and our concluding $\S 7$.

\subsection{Validation of the numerical scheme}

As a simple validation test we imposed the symmetric Jeffery-Hamel solution across both the inlet and the outlet of the domain as a Dirichlet condition:

$$
u=\frac{G^{\prime}(\phi)}{\alpha r}, \quad v=0 \quad \text { on } r=\eta^{-1}, 1,
$$

which led to good agreement with the similarity solution across the entire domain for all Reynolds numbers and channel angles considered. For example, for $R e=40$, $\alpha=0.2$ and $\eta=100$ the error in the radial velocity scaled by $r$ was found to be everywhere less than $2 \times 10^{-3}$ for a fixed coarse mesh of 32 by 16 elements. As expected for the chosen finite elements $\left(Q_{2} P_{-1}\right.$ see Gresho \& Sani 1998, p. 554) numerical error was found to decrease quadratically under uniform mesh refinement. Additional validation was performed by a detailed comparison of perturbations with the spatial (Dean) eigenmodes of the similarity solution, see $§ 6.2$. 


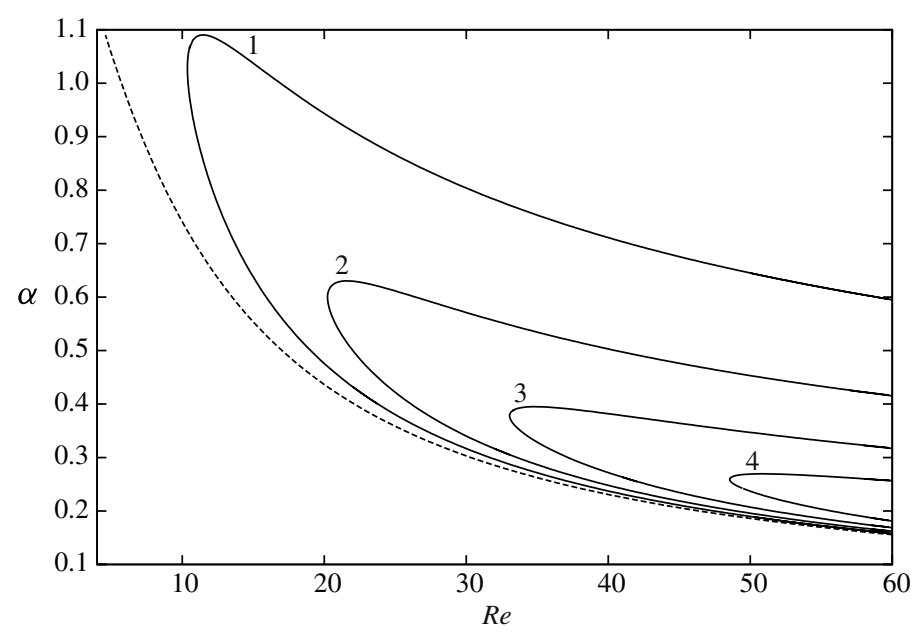

FIGURE 3. Numerically calculated loci of pitchfork bifurcations in the $(R e, \alpha)$ plane for a radius ratio of $\eta=100$, a quadratic inlet condition and a PTF outlet (solid lines). Each curve is associated with a temporally neutral, two-dimensional eigenmode that breaks the midplane symmetry of the flow and the numbering is for later reference. The dashed line indicates the location of the (infinite-domain) similarity solution pitchfork bifurcation, namely the set of critical values $\operatorname{Re}_{c}(\alpha)$ corresponding to boundary $\mathscr{B}_{2}$.

\section{The bifurcation structure in a finite domain}

In this section, all results are presented for the Dirichlet inlet boundary condition (2.4) and the PTF outlet boundary condition (2.3). For these conditions, the computational task is to locate pitchfork bifurcations, limit points and Hopf bifurcations in the $\alpha-R e$ parameter plane (at a fixed radius ratio $\eta$ ). These critical points are obtained by forming the appropriate extended system, which ensures that the solution lies exactly at the bifurcation point, see for example Salinger et al. (2002). Once a critical point has been located, its locus in the $\alpha-R e$ parameter plane can be determined by arc-length continuation of the extended system. In the following discussion we address each bifurcation type individually to construct a full picture of the solution structure across the parameter plane at fixed radius ratio $\eta$. We concentrate on the specific case of $\eta=100$, as a representative 'large' domain that could be plausibly realized experimentally, however results at this value are also qualitatively typical of those found over a broad range of radius ratios.

\subsection{The loci of symmetry-breaking pitchfork bifurcations}

Figure 3 shows the pitchfork bifurcations found for $\eta=100$ and $0 \leqslant R e \leqslant 60$. The loci of pitchfork bifurcations form a series of nested neutral curves in the $(R e, \alpha)$ plane. We conjecture that an infinite number of neutral curves can be found in the combined limit of $\alpha \ll 1, R e \gg 1$ and $\eta \gg 1$. However, for the range of parameter values we have considered $(0 \leqslant R e \leqslant 100)$ only six are present. At these boundaries the symmetric outflow state becomes unstable to midplane symmetrybreaking bifurcations.

In figure 4 we show streamlines for the critical eigenfunctions of the pitchfork bifurcations associated with the lower branches of the outer four neutral curves shown in figure 3. The eigenfunctions shown are for $\alpha=0.1$ and are associated with the continuation of the curves shown in figure 3 to somewhat larger Reynolds 
(a)

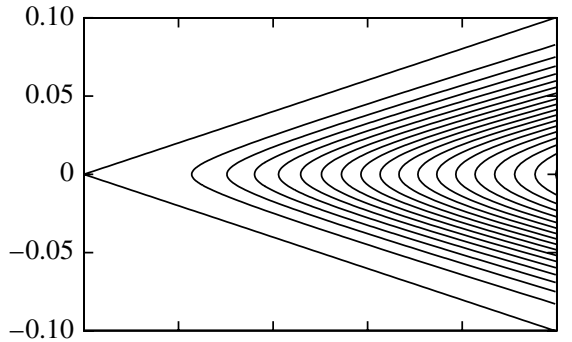

(c)

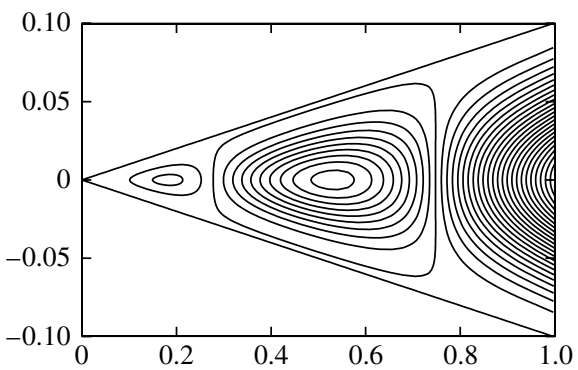

(b)

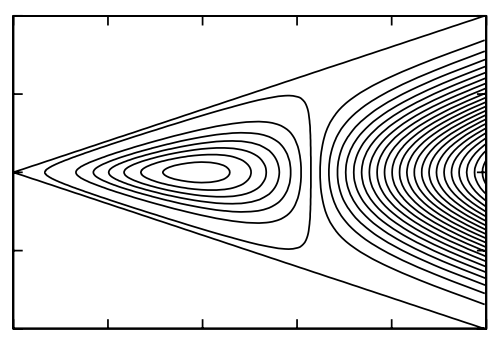

$(d)$

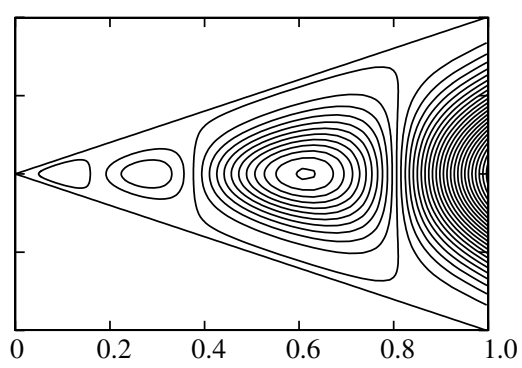

FIGURE 4. Streamlines of the temporally neutral, finite-domain, symmetry-breaking, eigenfunctions associated with the lower branch of the outer four neutral curves given in figure 3, with a wedge angle of $\alpha=0.1$ and radius ratio of $\eta=100$. The contour spacing is uniform. (a) Neutral curve 1, $\operatorname{Re}=94.7$; (b) neutral curve 2, $\operatorname{Re}=96.0$; (c) neutral curve 3, $\operatorname{Re}=97.9 ;(d)$ neutral curve $4, \operatorname{Re}=100.4$.

numbers. It can be seen that each successive neutral curve introduces one additional recirculation zone into the eigenfunction. The number of recirculation zones for a given eigenfunction being one less than the number of the associated neutral curve.

For a fixed value of $\alpha$, below the 'nose' of each neutral curve, we find that the pitchfork bifurcations shown in figure 3 are 'right facing', in the sense that the resulting symmetry-broken flows exist for $R e>\widehat{R e}_{c}(\alpha)$ in all cases. Here we introduce the hat notation, $\widehat{R e}_{c}$, to distinguish critical Reynolds numbers of the finite domain from that associated with the Jeffery-Hamel pitchfork bifurcation. Streamlines of the finite-amplitude state after bifurcation from the fourth neutral curve can be seen in figure 5 .

Figure 3 demonstrates that the lower branch of pitchfork bifurcations in a finite domain are in good quantitative agreement with the boundary $\mathscr{B}_{2}$ predicted by the infinite-domain similarity solution. That the similarity solution successfully predicts the critical Reynolds number of the finite-domain bifurcation is surprising, given that the finite-domain bifurcation is supercritical whereas the similarity solution bifurcation is subcritical at these points.

\subsection{The loci of limit points and Hopf bifurcations}

Using the same radius ratio $(\eta=100)$ and boundary conditions as for figure 3 , we determine the location of limit points associated with the symmetry-broken states and Hopf bifurcations that arise from the primary symmetric-outflow solutions. Figure 6 shows these results for the two outermost neutral curves, and we anticipate similar qualitative behaviour for each successive neutral curve. Clearly the first bifurcation is of the most practical relevance and we have made no attempt to obtain yet more boundaries at higher Reynolds numbers. An alternative representation of the behaviour 


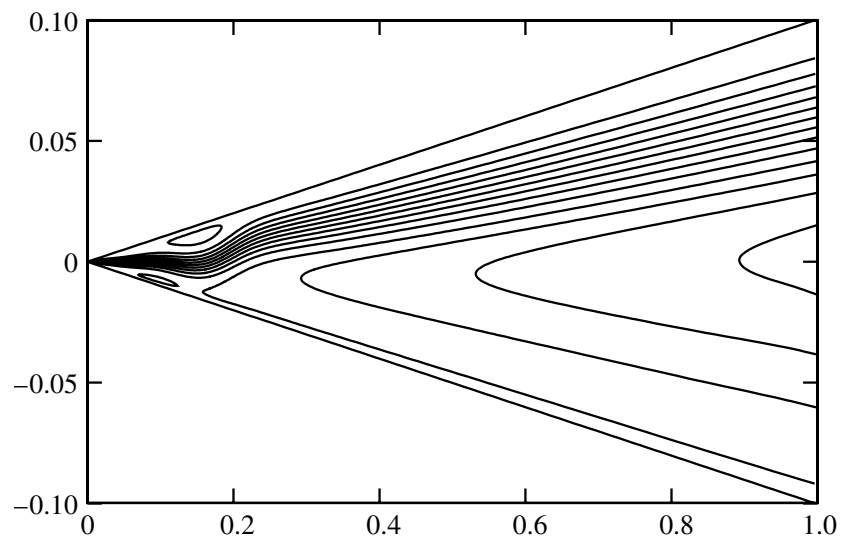

FIGURE 5. Streamlines of the symmetry-broken flow arising from the pitchfork bifurcation associated with the lower branch of the fourth neutral curve given in figure 3 at $\alpha=0.1$ and $\eta=100$. The bifurcated state is shown for $R e=120$, but the bifurcation is at $R e=100.4$. The contour spacing is 0.05 , however the contours near $\psi= \pm 0.5$ have been adjusted slightly in order to visualize two closed recirculation regions in this nonlinear flow state.

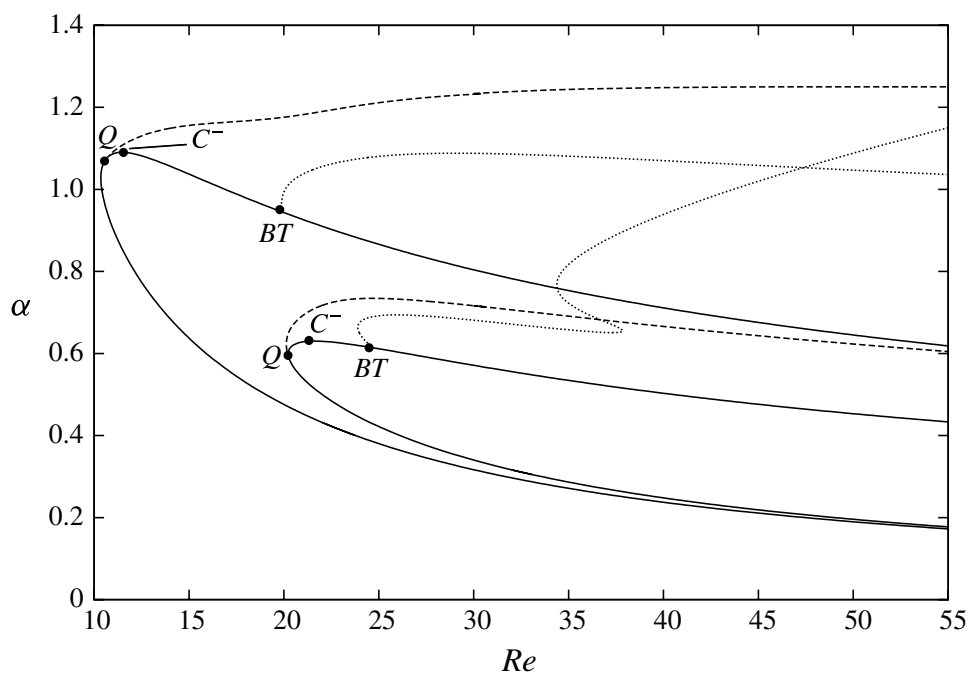

FIGURE 6. The location of pitchfork, limit-point and Hopf bifurcations in the $(R e, \alpha)$ plane. Only the outer two pitchfork bifurcation curves are shown (solid lines) along with the limit points of bifurcated (symmetry-broken) solutions (dashed lines) and the Hopf bifurcations associated with the base state (dotted lines). Here $C^{-}$denotes a coalescence point, $Q$ denotes a quartic point and $B T$ denotes a Bogdanov-Takens point. Results are for $\eta=100$, with a quadratic inlet profile and a PTF outlet.

shown in figure 6 is presented in figure 7, which shows schematic bifurcation diagrams (corresponding to the outermost neutral curve) that illustrate the solution structures that would be observed at different fixed values of $\alpha$ as $R e$ increases.

The Hopf bifurcations terminate with Bogdanov-Takens bifurcation points (Kuznetsov 1998), at which the period tends to infinity and they reconnect with the appropriate loci of pitchfork bifurcations. The first Hopf bifurcation is supercritical, 
(a)

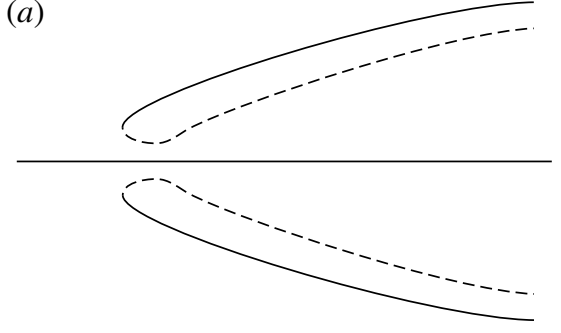

(c)

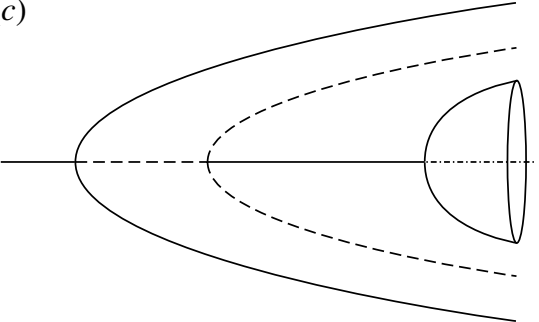

(b)

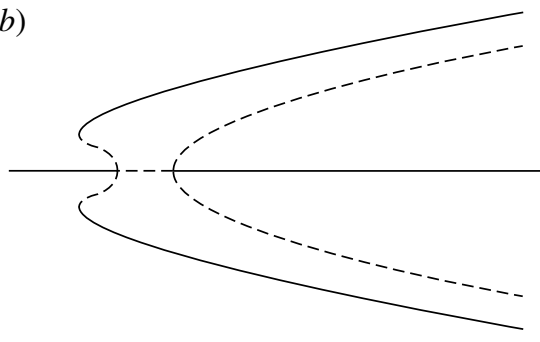

(d)

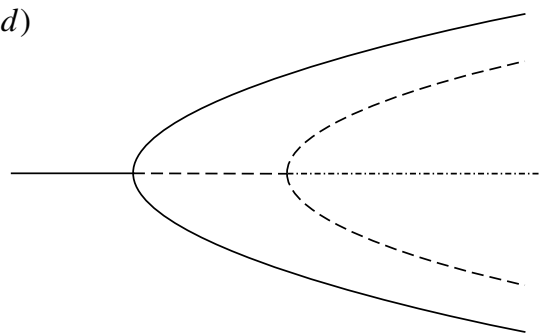

FIGURE 7. A schematic diagram of the bifurcated states associated only with the outermost neutral curve shown in figure 6 . Bifurcated states are shown here with a notional metric that reflects the degree of midplane asymmetry of the flow as the ordinate and with the Reynolds number as the control parameter (at fixed $\alpha$ and $\eta$ ), increasing from left to right. Solid lines indicate temporally stable solutions whilst dashed lines indicate solutions that are temporally unstable to at least one mode, as determined by two-dimensional eigenvalue calculations. The four schematic views are shown in order of decreasing $\alpha$. Their relation to figure 6 is as follows: (a) above $C^{-} ;(b)$ between $Q$ and $C^{-} ;(c)$ between $B T$ and $Q ;(d)$ beneath $B T$.

as inferred from a related initial-value problem, and it is worth noting that at values of $\alpha$ that lie above the Bogdanov-Takens point, the upper branch of the neutral curve restabilizes the symmetric base state, which then remains stable until the Hopf bifurcation is encountered at larger values of $R e$ (figure $7 c$ ).

The limit points connect to the pitchfork bifurcations at quartic points, where the criticality of the pitchfork changes, revealing the manner in which the pitchfork bifurcations cease to exist as the wedge angle is widened. For small values of $\alpha$, the upper/lower branches of a particular neutral curve are right-facing pitchfork bifurcations for increasing $\operatorname{Re}$ (figure $7 c$ and $d$ ). As $\alpha$ increases beyond values at which a limit point exists for $\operatorname{Re}<\widehat{R e}_{c}$ the first bifurcation changes to become leftfacing via a quartic point, leading to hysteresis, albeit over a very small parameter range (figure $7 b$ ). As $\alpha$ is increased further the two pitchfork bifurcations coalesce at a $C^{-}$coalescence point (Cliffe, Spence \& Tavener 2000, p. 48) and disappear as the associated bifurcated states detach from the base symmetric solution branch (figure $7 a$ ).

In general, therefore, the flow states at larger values of wedge-angle are more complicated, with non-uniqueness, either in the form of the coexistence of stable symmetric and asymmetric states or the coexistence of stable steady and periodic asymmetric states.

\section{Isolated steady solution branches}

In the previous section, we explored the solution structure using continuation methods, which only give information about connected solutions. In this section, we 
describe alternative states that are isolated from those described above. As in $\S 3$ all results are presented for the Dirichlet inlet boundary condition (2.4) and the PTF outlet boundary condition (2.3).

At a separation angle of $\alpha=0.1$ the first pitchfork bifurcation occurs at $\widehat{R e}_{c} \approx 94.72$. Unsteady computations at this channel angle are entirely in line with the (temporal) eigenvalues obtained for the global problem, showing that the symmetric base flow branch is stable to sufficiently small perturbations for $\operatorname{Re}<\widehat{\operatorname{Re}}_{c}$. For $\operatorname{Re}>\widehat{\operatorname{Re}}_{c}$ the symmetric solution is indeed found to be unstable. However, although the bifurcation at $\widehat{R e}_{c}$ is supercritical, with (locally) stable symmetry-broken states present for $R e>\widehat{R e}_{c}$, these were not always the states that were achieved in the large time limit. Our numerical results indicate that the eventual state of the unsteady system depends sensitively on the initial conditions in this parameter regime; only some computations converged to the symmetry-broken state that arises from the pitchfork bifurcation at $\widehat{R e}_{c}$.

By performing unsteady computations for the particular values $\alpha=0.1$ and $\eta=100$ we were able to identify two new distinct steady solutions for $R e \leqslant 120$. These states are first located (approximately) via an initial-value calculation evolved to relatively large times. This approximate solution is then used as the initial guess for the iterative solution of the steady equations, the resulting steady solution is then continued to other values of $R e$ at fixed $\alpha$ and $\eta$. In both cases the new solutions were found to remain disconnected from the bifurcation structure described in the previous section.

In order to distinguish between the various symmetry-broken flows we introduce a global measure of flow asymmetry, $S=S_{-}+S_{+}$, where $S_{ \pm}$are integrals along the bounding planes:

$$
S_{ \pm}=\left.\int_{\eta^{-1}}^{1} \frac{\partial u}{\partial \phi}\right|_{\phi= \pm 1} \mathrm{~d} r .
$$

The two isolated (steady) solution branches are shown in figure 8 which depicts one half of the bifurcation diagram, the full picture being obtained by a reflection in the line $S=0$. Both solution branches consist of a stable and an unstable part connected by a limit point at the minimum value of the Reynolds number for which they exist. We denote the first isolated solution branch by B1 and the second new branch by B2.

Inset into figure 8 are depictions of the streamlines associated with temporally stable solutions on each of the two new branches, B1 and B2. Solutions on these isolated branches are characterized by regions of strong jet-like outflow adjacent to one wall, with the jet alternating from wall to wall as it develops down the channel. For example, for $R e=120$, a B2 temporally stable state has outflow concentrated adjacent to $\phi=-1$ for $0.1<r<0.3$ and adjacent to $\phi=1$ for $0.5<r<0.8$. These regions are connected by a zone of rapid adjustment in which the jet switches from one side of the wedge to the other. These qualitative features are common to all of the disconnected solutions discussed here and the adjustment zones become (radially) smaller as the Reynolds number is increased. The relationship between these solutions and those of Kerswell et al. (2004) is discussed in $\S 4.2$.

\subsection{Limit-point boundaries for the isolated branches}

In the parameter regime spanned by figure 8 there is non-uniqueness of temporally stable solution branches, with up to six potential solutions (three solutions and their reflections in the midplane); and the precise solution achieved in any given initialvalue computation depends upon the initial conditions for the flow. The number of 


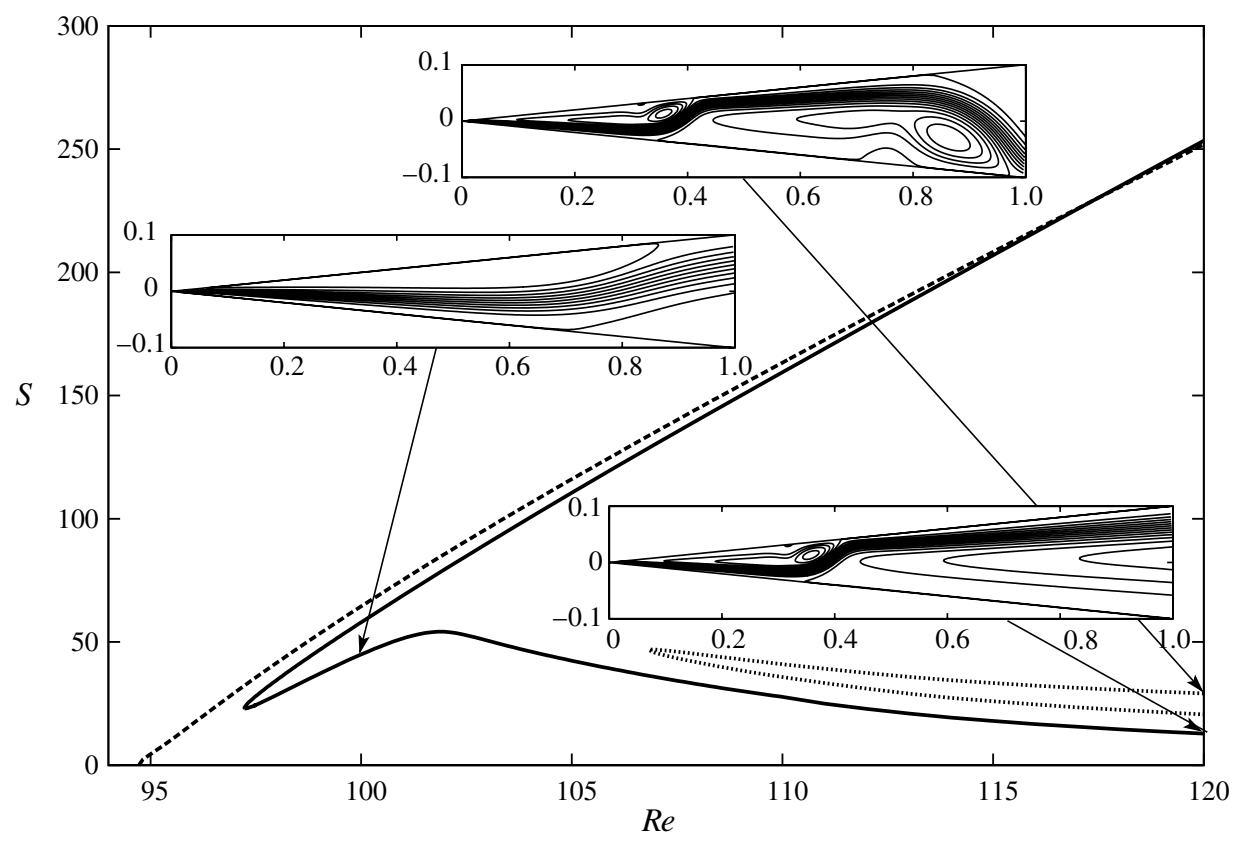

FIGURE 8. Isolated solution branches B1 (solid) and B2 (dotted) for the finite-domain problem subject to the quadratic inlet condition and a PTF outlet; the metric $S$ is defined by (4.1). The symmetry-broken solution branch that arises from the first finite-domain pitchfork is shown as the dashed line. The solutions shown are for a wedge angle $\alpha=0.1$ and radius ratio $\eta=100$. The inset figures depict streamlines for the stable (lower) branch of B1 at $R e=100$ and $R e=120$ and for the stable (upper) branch of $\mathrm{B} 2$ at $R e=120$.

available steady solutions depends upon the location of the limit point of the solution B1 (and obviously B2) as a function of the channel divergence angle $\alpha$.

Given a solution on the isolated branch B1 we can determine the minimum Reynolds number for its existence (at fixed $\alpha$ and $\eta$ ) by forming the extended system associated with the limit point. The locus of this limit point in the $\alpha-R e$ plane can then be determined via arc-length continuation in these two parameters at fixed radius ratio $\eta$. By this procedure we obtain the critical boundary of figure $9(a)$, which shows the variation of the Reynolds number at which the limit point of isolated solutions exists for varying channel angle $\alpha$. As the channel divergence increases, a global lower bound of $R e \approx 40$ is found for the existence of the isolated solution B1 when $\eta=100$. Computation of the solution B1 at a fixed angle of $\alpha=0.28$ and $\eta=100$ indicates that, on decreasing $\alpha$ the solution first appears as an isola, as shown in figure $9(b)$.

\subsection{The relationship to the steady waves of Kerswell et al. (2004)}

The solutions on the temporally stable portion of branch B2 possess a spatially wavelike radial structure. Similar solutions with regions of wall-adjacent outflow connected by regions of rapid adjustment have been discussed before by Tutty (1996) and Kerswell et al. (2004). Tutty computed solutions in both symmetric and asymmetric expanding channels with extremely large radius ratios and observed the existence of a spatial wave, periodic along the channel in his (logarithmically spaced) computational coordinate. Concentrating on parameter values near $\mathscr{B}_{2}$ he was able to observe the wave for values of the Reynolds number both greater than, and slightly less than $R e_{c}$ 

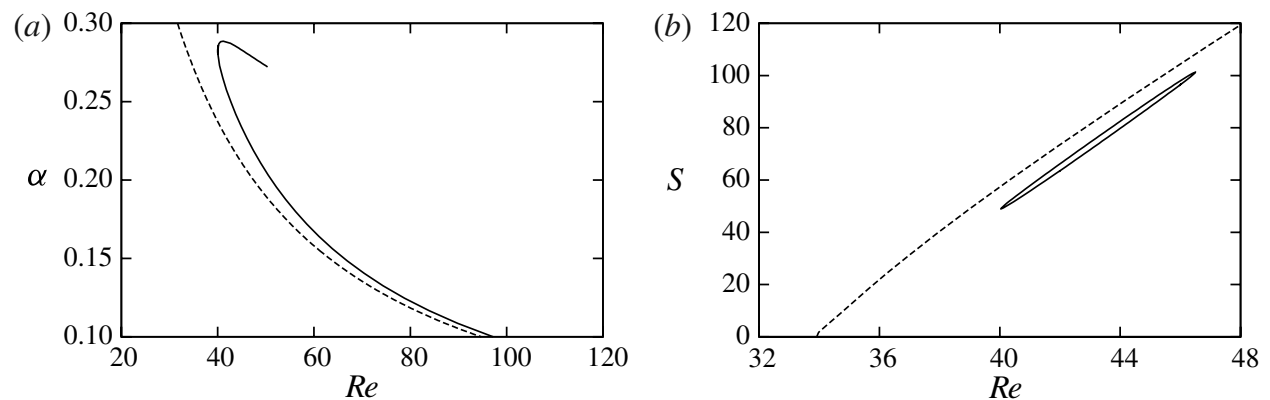

FIgURE 9. (a) The locus of limit points for the isolated solution branch B1 (solid line) with $\eta=100$. The dashed line indicates the 'first' locus of pitchfork bifurcations for the symmetric outflow solution, that is, neutral curve 1 in figure 3 . No attempt was made to continue the second limit point past $R e=50$. (b) The metric $S$, (4.1), for the isolated branch B1 at a larger divergence angle of $\alpha=0.28$ (solid line). The dashed line shows the same metric for the symmetry-broken states that arise from the pitchfork bifurcation of neutral curve 1 .

(the location of the pitchfork bifurcation in the Jeffery-Hamel similarity solution). For $R e<R e_{c}$ the existence of the spatial wave was dependent upon the inlet geometry, relying on the existence of a sudden expansion step of order one

Motivated by the results of Tutty, Kerswell et al. (2004) obtained solutions to the steady Navier-Stokes equations using the computational coordinates,

$$
X=\omega \log \left(\frac{r}{r_{1}}\right), \quad Y=\phi,
$$

where $\omega$ represents a wavenumber and $r_{1}$ is a constant phase shift. They employed periodic boundary conditions with respect to variations in the $X$ coordinate, and allowed $\omega$ to vary in order to locate solutions satisfying the symmetry condition, $\psi(X+\pi,-Y)=-\psi(X, Y)$, over the range $0 \leqslant X \leqslant 2 \pi$. In this manner the spatial wave solution observed by Tutty was obtained directly for infinite domains. This solution was found to persist beneath $\mathscr{B}_{2}$ where it was observed to reconnect with the similarity solution via a heteroclinic orbit across the arms of the Jeffery-Hamel pitchfork bifurcation.

Although the structures observed here are not exactly periodic we conjecture that they are the finite-domain realization of the logarithmically spaced waveform of Kerswell et al. (2004). Tutty (1996), in contrast, was able to observe an almost exactly periodic waveform in a finite domain, however the radius ratio required was orders of magnitude larger than considered herein. Figure 10 shows solutions for $R e=200.408, \alpha=0.05003$ and for radius ratios $\eta=100$ and $\eta=1000$. Increasing the radius ratio of our domain to $\eta=1000$ did allow us to recover solutions with a larger number of adjustment regions, as illustrated in figure 10. Nevertheless, in any physical experimental investigation it seems highly unlikely that such extremely large radius ratios could be realized effectively.

For the parameter values used to produce figure 10, Kerswell et al. (2004) found a periodic solution with a wavenumber of $\omega=4.1691$. In figure 11 we present a part of the solution of figure $10(b)$ but using the coordinates (4.2) with $\omega=4.1691$. There is good qualitative agreement between figures 11 and $8(b)$ of Kerswell et al. (2004), supporting our conjecture that the steady nonlinear wave solutions of Kerswell et al. 

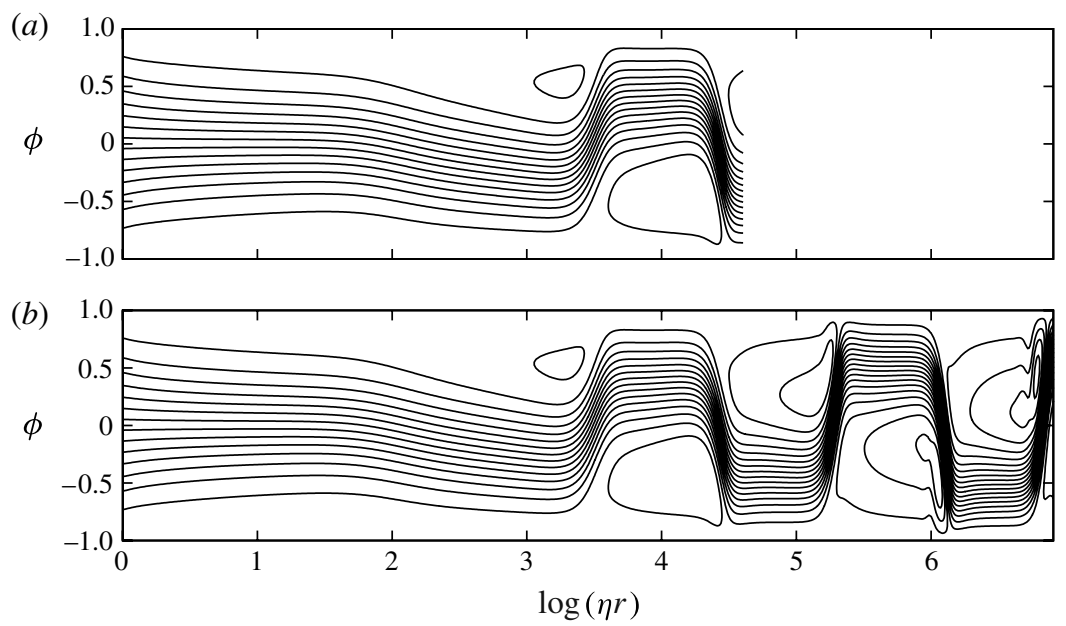

FIGURE 10. Streamlines for flow in the finite domain for $R e=200.408$ and a wedge angle of $\alpha=0.05003$. The boundary conditions are a quadratic inlet profile and a PTF outlet. The contour spacing is 0.07 . The radius ratio in each case is $(a) \eta=100$ and $(b) \eta=1000$.

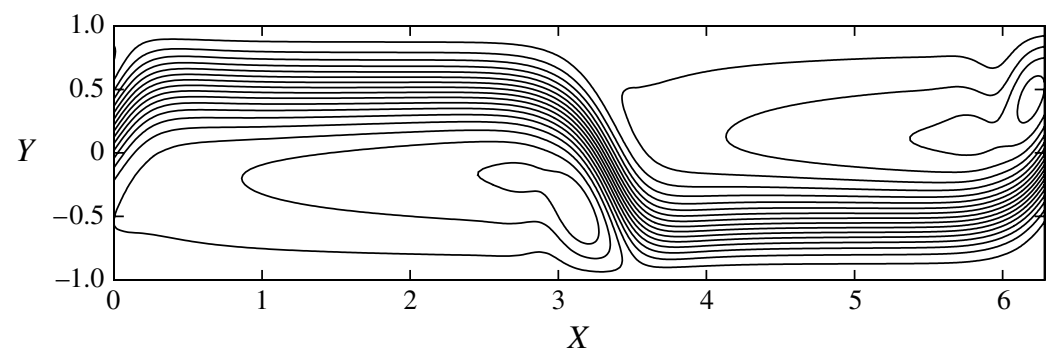

FIGURE 11. Streamlines of the solution for $R e=200.408, \alpha=0.05003$ and $\eta=1000$. The solution is shown in the computational coordinates (4.2) used by Kerswell et al. (2004) and using the same scaling factor, $\omega=4$.1691. A single wavelength (near to the outlet) is shown for comparison with figure $8(b)$ of Kerswell et al. (2004). The boundary conditions are a quadratic inlet and a PTF outlet.

(2004) persist for finite (but in some sense 'large') domains, but become disconnected in the bifurcation structure.

\section{The physical relevance of our numerical results}

In the previous sections, we have presented a comprehensive picture of the solution structure for flow in a diverging channel for a specific choice of boundary conditions. For these results to be of practical relevance we require that: (i) the solutions are stable; (ii) the domain is experimentally realizable; and (iii) there is no strong sensitivity to inlet/outlet constraints.

The first (outermost) of the nested neutral curves shown in figure 3 , is of most physical significance because the underlying symmetric base state will already be unstable when encountering the subsequent curves. For this reason we have concentrated on the bifurcation structure associated with this first instability in figures 6-9. Eigenvalue computations (and unsteady computations) have demonstrated 


$\begin{array}{lccc}\alpha & \text { Jeffery-Hamel } & \text { Quadratic } & \text { Plug flow } \\ 0.1 & 94.18 & 94.72 & 94.86 \\ 0.4 & 23.27 & 23.77 & 23.90 \\ 0.7 & 12.85 & 13.72 & 13.98\end{array}$

TABLE 1. The variation of the critical Reynolds number of the first symmetry-breaking bifurcation in a finite domain with radius ratio $\eta=100$ and inlet boundary conditions given by (2.4) 'Quadratic', (5.1) 'Jeffery-Hamel' and (5.2) 'Plug flow'. The outlet boundary condition is PTF in each case.

that the first bifurcation is supercritical and that the nonlinear symmetry-broken state that develops is stable. We therefore anticipate that this is a state that will be observable in an experiment provided that three-dimensional effects are not important.

However, the unsteady computations described in $\S 4$ have demonstrated nonuniqueness in possible stable states, with the system evolving to either the aforementioned bifurcated solution or an isolated spatial-wave solution. Although we do not attempt to give a complete description of the basin of attraction of these states we note that the isolated steady states were readily obtained in our initialvalue computations. We anticipate that both types of steady state will coexist in a corresponding experimental configuration; under the shared assumption that they are not unstable to three-dimensional modes. The critical boundaries beyond which this multiplicity in stable solutions exists are shown in figure $9(a)$ and present obvious quantities for experimental measurement. Herein we have chosen a radius ratio of $\eta=100$ as a value that is sufficiently large to allow the coexistence of the spatial-wave (isolated) states and the supercritically bifurcated states, but still remains a plausible geometry for a laboratory configuration.

Finally, we recognize that in an experimental configuration one has limited control of the inlet/outlet conditions. To examine the influence of these choices we have examined other inlet profiles; namely, the Jeffery-Hamel similarity solution

$$
u\left(r=\eta^{-1}, \phi\right)=\frac{\eta}{\alpha} G^{\prime}(\phi), \quad v\left(r=\eta^{-1}, \phi\right)=0,
$$

and another alternative

$$
u\left(r=\eta^{-1}, \phi\right)=\frac{\lambda \eta}{2 \alpha}\left(\frac{\cosh \lambda-\cosh \lambda \phi}{\lambda \cosh \lambda-\sinh \lambda}\right), \quad v\left(r=\eta^{-1}, \phi\right)=0,
$$

which, for large values of $\lambda$, is a 'plug flow' of $u \approx \eta(2 \alpha)^{-1}$ across the bulk of the range, with two thin regions near $\phi= \pm 1$ in which the profile adjusts to the no-slip condition. We choose $\lambda=10$ in this example.

The choices (2.4), (5.1) and (5.2) give three very different inlet profiles, yet for $\eta=100$ their influence on the finite-domain base flow is rather minimal; adjusting the inlet condition slightly alters the distance from the inlet at which an approximately self-similar flow first develops. A sensitive global measure of the symmetric outflow state is the location of the first symmetry-breaking bifurcation, and in table 1 we show the variation of this quantity for all three choices of inlet condition. We observe that the (large) variations in the inlet profile leads to a variation of less than $1 \%$ in the critical Reynolds number when $\alpha=0.1$ and $\eta=100$. The influence of the inlet profile is stronger for wider expansion angles: the change in critical Reynolds number is approximately $8 \%$ at $\alpha=0.7$. Hence, we should expect good agreement 
with experimental data overall, but particularly robust agreement for small channel divergence angles. The solution structure is even less sensitive to outflow conditions.

We should note that it is possible to change the finite-domain solution significantly by choosing very specialized inlet constraints. For example, if we apply pseudotraction conditions that are consistent with the infinite-domain Jeffery-Hamel solution at both the inlet and the outlet (see Haines 2010; also Hewitt \& Hazel 2007 for details of an equivalent formulation appropriate to rotating-disc flow) then this solution is also a solution of the finite-domain problem. In this case Jeffery-Hamel flow, including its subcritical bifurcation at $R e_{c}$, is accurately resolved in a finite domain by our numerical scheme. Nevertheless, for general choices of inlet/outlet constraints, we find a supercritical pitchfork bifurcation at the loss of midplane symmetry, which we view as the generic and experimentally relevant behaviour.

We have also investigated a related geometry in which long parallel inlet/outlet channels are included, in order to somewhat decouple the bulk of the domain from the choice of inlet/outlet conditions. We recover the same qualitative behaviour that is described herein, again with particularly good quantitative agreement at low divergence angles.

The experimental study of Putkaradze \& Vorobieff (2006) examined the flow in a wedge (typically) of semi-angle 0.28 radians and radius ratio approximately equal to 29. They confirmed that the pure outflow solution is stable and, furthermore, that it is always obtained for $R e<R e_{c}$. For $R e>R e_{c}$ they report both symmetric and (as predicted herein) symmetry-broken stable solutions; however they also report an accompanying hysteresis (which we do not find in our numerical results). Recently Yu, Shao \& Lin (2010) have presented numerical computations designed to replicate the global experimental configuration of Putkaradze \& Vorobieff (2006). They suggest that the apparent hysteresis might actually be a transient behaviour that is slow relative to the observation time of the experiment. Further experimental work is clearly warranted to investigate these effects and to examine the predicted coexistence of states.

\section{The relevance of the Jeffery-Hamel similarity solution to finite domains}

From our investigation of the finite-domain computational problem we conclude that, apart from a specialized case designed to reproduce the similarity-solution behaviour as mentioned above, the choice of inlet/outlet conditions has a limited impact on the solution. Furthermore, there is good agreement between the type $\mathrm{II}_{1}$ or $\mathrm{II}_{2}$ self-similar solution and the symmetric finite-domain solution over the bulk of the domain.

Perhaps the most surprising feature is that the finite-domain midplane-symmetric flow has a sequence of pitchfork bifurcations and that, in the limit of $\operatorname{Re} \gg 1$ and $\alpha \ll 1$, these bifurcations approach the single critical value obtained from the selfsimilar solution. The agreement is surprising because the predicted criticality of these bifurcations is incorrect, as is the number of such bifurcations. In this section we explain this apparent discrepancy by means of a discussion of the spatial eigenmodes of the Jeffery-Hamel states and, in doing so, demonstrate how the self-similar solution is relevant to finite-domain flows.

\subsection{Spatial stability of Jeffery-Hamel states}

Following Dean (1934) we consider the effect of a perturbation to the dimensional streamfunction of the form,

$$
\psi=Q\left(G(\phi)+\epsilon \hat{r}^{\lambda} \tilde{g}(\phi)\right) .
$$




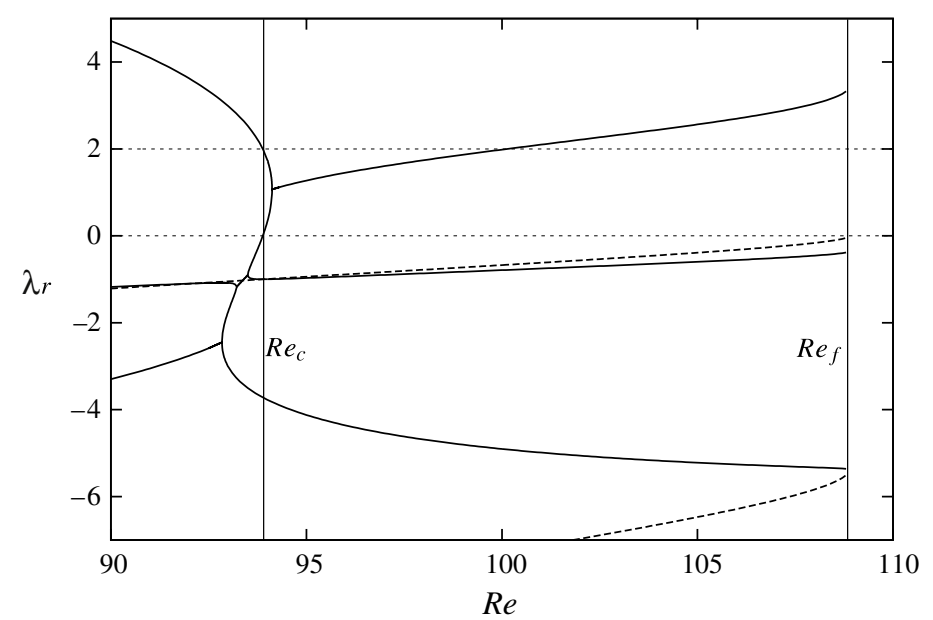

FIGURE 12. The real part of the dominant spatial eigenvalues of the Jeffery-Hamel solution for $\alpha=0.1$. The eigenvalues passing through 0 and 2 at $R e_{c}=93.8851$ are indicated by a vertical line. An eigenvalue corresponding to a symmetric eigenvector can also be seen approaching $\lambda=0$ as $R e \rightarrow R e_{f}=108.8864$, as defined in figure 1 .

On linearizing for small $\epsilon$ we obtain the following quartic eigenproblem for $\lambda$,

$$
\begin{aligned}
& \alpha \operatorname{Re}\left((\lambda-2) G^{\prime} \tilde{g}^{\prime \prime}-2 G^{\prime \prime} \tilde{g}^{\prime}-\lambda G^{\prime \prime \prime} \tilde{g}\right)+\alpha^{3} \operatorname{Re} \lambda^{2}(\lambda-2) G^{\prime} \tilde{g} \\
& \quad=\tilde{g}^{(i v)}+\alpha^{2}\left((\lambda-2)^{2}+\lambda^{2}\right) \tilde{g}^{\prime \prime}+\alpha^{4} \lambda^{2}(\lambda-2)^{2} \tilde{g},
\end{aligned}
$$

together with the boundary conditions,

$$
\tilde{g}( \pm 1)=0, \quad \tilde{g}^{\prime}( \pm 1)=0,
$$

specifying no-slip and impermeability at the wedge walls.

We have solved (6.2) using a standard Runge-Kutta method. In order to ensure that no eigenvalues are overlooked, initial approximate values for all eigenvalues $\lambda$ are found using a standard global method (QZ) applied to a second-order accurate discretized version of the eigenproblem. Figure 12 shows the real part of $\lambda=\lambda_{r}+\mathrm{i} \lambda_{i}$ at the representative value of $\alpha=0.1$. At the pitchfork bifurcation, $R e=R e_{c}$ there are eigenvalues of zero and two. Banks et al. (1988) were able to show that the eigenvector corresponding to both these eigenvalues is $\tilde{g}=G^{\prime}(\phi)$. It can also be seen in figure 12 that eigenvalues for which $0<\lambda_{r}<2$ are found only for $R e>R e_{c}$, as conjectured by Banks et al. (1988). The eigenvectors associated with the $\lambda=0,2$ eigenvalues (at $R e=R e_{c}$ ) have an opposite symmetry to the base flow. We shall refer to such eigenvectors as antisymmetric, whilst those sharing the symmetry of the base flow will be termed symmetric.

Banks et al. (1988) further conjectured the existence of eigenvalues $\lambda=0$ and $\lambda=2$ at the turning point boundary, denoted $\mathscr{B}_{3}$ by Fraenkel (1962). However, although an eigenvalue (corresponding to a symmetric eigenvector) is visible in figure 12 approaching zero as the Reynolds number approaches the turning point, we have not observed an eigenvector with $\lambda=2$ there.

Figure 12 can be compared with figure 13 of Goldshtik et al. (1991) which gives results for $\alpha=0.0873$. The qualitative behaviour is in broad agreement. However Goldshtik et al. do not appear to have correctly captured the behaviour of the first of 
the two eigenmodes,

$$
\tilde{g}=\cos (\alpha \phi) G^{\prime}(\phi) \quad \text { and } \quad \tilde{g}=\sin (\alpha \phi) G^{\prime}(\phi),
$$

shown by Drazin (1995) to exist for $R e=R e_{c}$ and $\lambda=-1$. A small complex conjugate region nearby is also missing.

Figure 12 shows eigenvalues with both negative and positive real parts for Reynolds numbers less than $R e_{c}$. However, the existence of eigenmodes that grow towards $\hat{r}=0$ and $\hat{r}=\infty$ does not necessarily indicate spatial instability. In fact, for $R e=0$ two infinite families of eigenvectors are known to exist corresponding to eigenvalues of either sign (Banks et al. 1988); the spectrum is symmetric about $\lambda=1$ when $R e=0$. These two families contain both symmetric and antisymmetric eigenvectors and Banks et al. (1988) suggest that each family represents a complete set of eigenfunctions. Thus in order to determine the evolution of a perturbation uniquely in either the 'downstream' or 'upstream' direction it must be the case that only one of the two infinite families of eigenfunctions is appropriate "so that the perturbation may be independently specified for $-\alpha \leqslant \theta \leqslant \alpha$ at (two distinct values of $\hat{r}$ )" (Banks et al. 1988).

The concept of spatial stability is not entirely straightforward in this context. One would normally appeal to a discussion of the group velocity of the disturbance to decide whether a disturbance can propagate energy in the direction in which spatial growth can be achieved. However, in the context of Dean modes, the inclusion of the unsteady terms breaks the self-similar separation. One may view the problem in another way when considering spatial stability with respect to growth in either the downstream (to large $\hat{r}$ ) or upstream (to small $\hat{r}$ ) directions. Having chosen a direction (either upstream or downstream) we shall refer to the (countably infinite family of) eigenmodes that influence the response in that direction as the set of 'relevant modes', whilst those that have no bearing are 'irrelevant modes'.

In the absence of unsteadiness we identify the relevant and irrelevant modes in each case in such a way that the flow is necessarily (spatially) stable for $R e=0$. Thus the relevant modes when considering downstream propagation are those for which $\lambda_{r}<0$ at $R e=0$ whilst the relevant modes for upstream propagation are those for which $\lambda_{r}>2$ at $R e=0$. Continuous variation of the Reynolds number then indicates that the flow will remain spatially stable to linear perturbations in both directions until such time as one of the eigenvalues crosses the imaginary axis. That is, until a mode that is relevant to the discussion of either up or downstream stability ceases to decay and begins to grow in the direction under consideration. In the context of the finite-domain (elliptic) problem it is a simple matter to check this interpretation.

\subsection{Realization of Dean modes in a finite domain}

Dennis et al. (1997) attempted to observe the role of Dean eigenmodes by imposing either a symmetric or an antisymmetric perturbation at a boundary of a finite computational domain. However, their attempt to recover the Dean modes resulted in limited success owing to computational restrictions and small domain sizes.

Given a finite domain we begin by imposing the similarity solution plus a small perturbation $(\tilde{u}, \tilde{v})$ as a Dirichlet condition at one end of our domain, that is

$$
u(r, \phi)=\frac{G^{\prime}}{\alpha r}+\frac{A}{\alpha} r^{\lambda-1} \tilde{g}^{\prime}(\phi), \quad v(r, \phi)=-A \lambda r^{\lambda-1} \tilde{g}(\phi),
$$

at either $r=\eta^{-1}$ or $r=1$. Here $\tilde{g}(\phi)$ is a Dean eigenmode that satisfies (6.2), and $G(\phi)$ is the Jeffery-Hamel outflow state. At the other end of the domain, a traction 


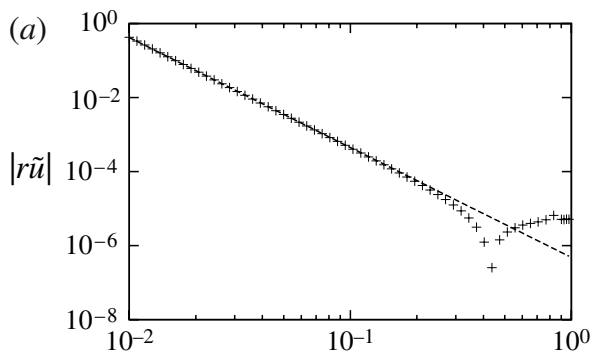

(b)
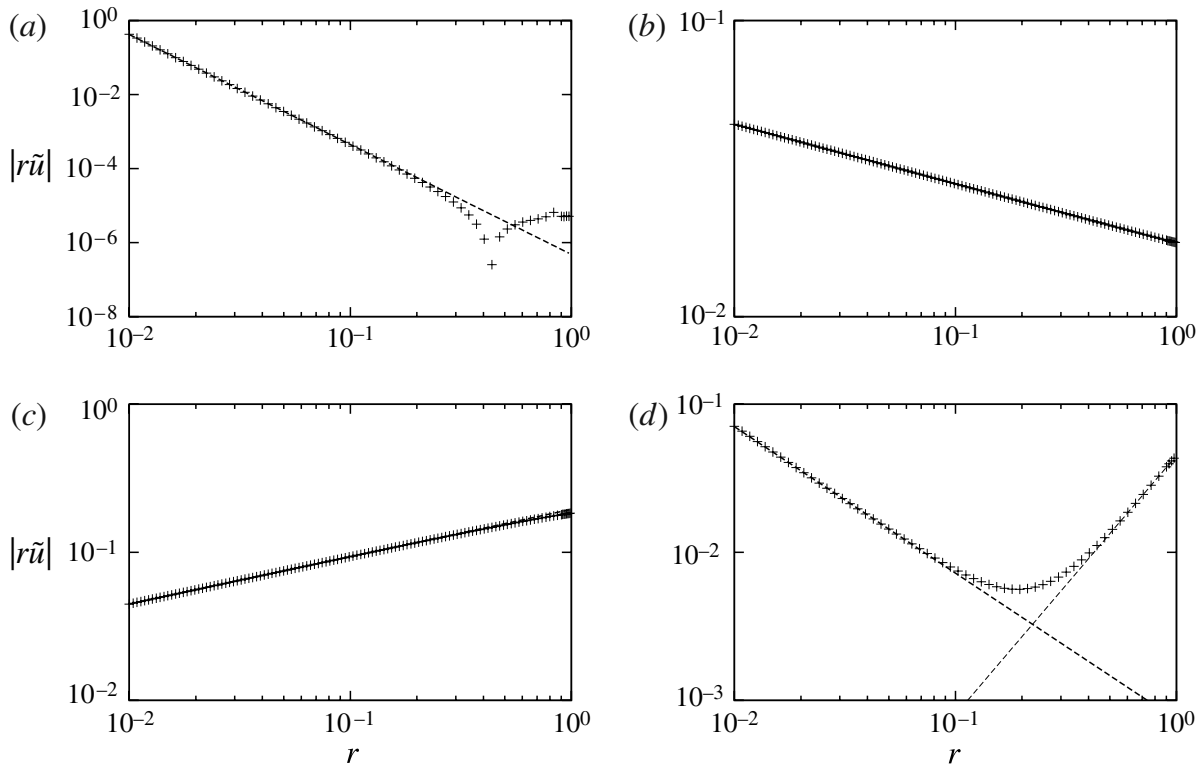

FIGURE 13. Radial slices (at constant $\phi$ ) showing the spatial development of perturbations applied at either the inlet or outlet of a finite domain for which the underlying base flow is of self-similar form. The perturbation is applied at only one end of the domain, whilst at the other we impose a pseudo-traction consistent with the similarity solution. The error from the similarity solution is shown on a log scale (individual points) together with the predicted decay rate provided by the spatial stability analysis of the similarity solution (dashed line). (a) $R e=60, \lambda=-2.97$ antisymmetric mode imposed at the inlet. (b) $R e=93.8, \lambda=-0.20$ antisymmetric mode imposed at the inlet. (c) $R e=94, \lambda=0.32$ antisymmetric mode imposed at the inlet. $(d) R e=94, \lambda=1.75$ antisymmetric mode imposed at the outlet. In all cases $\alpha=0.1, \eta=100$ and the radial slices are at $\phi=-0.5$.

condition consistent with the similarity solution is imposed, see Haines (2010). The amplitude coefficient $A$ is chosen so that the (relative) peak deviation from the selfsimilar flow remains below $5 \%$ across the computational domain. Once a solution has been found, we subtract the self-similar base flow solution from the resulting velocity field to define a two-dimensional perturbation to the radial velocity:

$$
\tilde{u}(r, \phi)=u(r, \phi)-\frac{G^{\prime}(\phi)}{\alpha r} .
$$

In figure 13 we present $r \tilde{u}(r, \phi=-0.5)$ and overlay the predicted algebraic (spatial) decay of the corresponding Dean eigenmodes, as determined from the spatial eigenvalue analysis. In all four cases we recover very good agreement.

The results shown in figure $13(a)$ are for an antisymmetric perturbation to the inlet at a Reynolds number $(\operatorname{Re}=60)$ that is significantly below the critical value $\left(R e_{c}=93.8851\right)$; this shows a clear decay from the inlet at the predicted rate until the finite-domain perturbation reaches the numerical error of the spatial discretization, or order $10^{-4}$ in this test case. In contrast figure $13(b, c)$ show the behaviour of antisymmetric perturbations for values of the Reynolds number that bracket the critical value. As the Reynolds number increases through $R e_{c}$ the critical eigenmode begins to grow away from the inlet rather than decay. 
Finally, figure $13(d)$ shows a perturbation imposed at the outlet, which again decays in agreement with the predicted value of $\lambda=1.75$. In this case, a numerical error is also observed to decay away from the inlet. The decay rate of this error is in accordance with the least stable symmetric mode, $\lambda=-0.99$. In all cases considered the azimuthal dependence of the finite-domain perturbation and that of the appropriate Dean mode were also found to be in good agreement.

The eigenmodes considered in figure 13 are all imposed at boundaries where they are considered to be relevant when considering growth into the interior of the domain. If a 'downstream relevant' eigenmode was imposed at the outlet, then it was observed to decay 'upstream' in agreement with a decomposition in terms of 'upstream relevant' modes, with the decay rate rapidly becoming that of the least stable relevant mode. The same behaviour was observed when imposing 'upstream relevant' modes at the inlet. This excellent agreement between the spatial stability analysis of the similarity solution and the two-dimensional, nonlinear, finite-domain computations gives us confidence that not only is our numerical scheme robust and accurate, but that our interpretation of 'spatial instability' is in quantitative agreement with the finite-domain problem.

As shown in figure 12 , there is another critical Reynolds number at which a spatially unstable 'downstream' (real) Dean mode combines with an 'upstream' (real) mode to give a complex conjugate pair. At this point the interpretation becomes more difficult and this complex Dean mode is better viewed as being in some sense 'global'. As we show below, it is this mode that will allow us to connect the finite-domain numerical results with the (infinite-domain) similarity solution.

\subsection{The connection to two-dimensional eigenmodes in the $\alpha \ll 1$ limit}

A key point to note arises from the results of figure 12. We know from the work of Banks et al. (1988) that at $R e=R e_{c}, \lambda=0$ and $\lambda=2$ are both eigenvalues, with the same associated eigenfunction $\tilde{g}=G^{\prime}(\phi)$. Similarly, in the $\alpha \ll 1$ limit, there is a 'Drazin' mode with $\lambda=-1$ and $\tilde{g} \approx G^{\prime}(\phi)$.

An asymptotic investigation of the Dean eigenvalue problem (outlined in Appendix) for $\alpha \ll 1$ reveals that, the $\lambda=0,2$ eigenvalues vary rapidly, combining to produce a complex conjugate pair when $R e-R e_{c}=O(\alpha)$. Once the pair becomes complex, then $\lambda_{i}$ increases steadily over this same (small) $R e-R e_{c}=O(\alpha)$ regime, however the eigenmode $\tilde{g}$ does not vary significantly, remaining $\tilde{g}=G^{\prime}(\phi)$ to leading order. As a consequence of these scalings, it is possible to find a (spatially) unstable eigenmode with $\lambda$ complex within a small $O(\alpha)$ region of the critical boundary $\mathscr{B}_{2}$ when $\alpha \ll 1$.

These simple observations are sufficient to allow us to construct a leading-order approximation to the two-dimensional eigenmode of the finite domain as a linear sum of a small number of Dean modes appropriate to an infinite domain. Furthermore, this approximation in terms of Dean modes explains why there is a sequence of bifurcations in the finite domain (rather than just one), and why their criticality differs from the infinite-domain Jeffery-Hamel prediction even though they seem to occur at the predicted critical Reynolds number.

For the purposes of explaining this decomposition, the simplest case to consider is that of a finite-domain flow with Dirichlet conditions applied at both the inlet and outlet. At both boundaries we prescribe the appropriate Jeffery-Hamel solution, therefore a finite-domain solution exists that is a type-II similarity solution everywhere in the channel. In finite domains, the imposition of boundary conditions at particular values of $r$ leads to the complex Dean modes being of much greater significance than 
the real Dean modes, however we know from the scalings presented in Appendix that such modes exist within an asymptotically small region around the boundary $\mathscr{B}_{2}$.

For a Jeffery-Hamel base flow solution, the $n$th Dean mode, with eigenvalue $\lambda_{n}=\lambda_{n r}+\mathrm{i} \lambda_{n i}$ and eigenfunction $\tilde{g}_{n}(\phi)=\tilde{g}_{n r}(\phi)+\mathrm{i} \tilde{g}_{n i}(\phi)$, leads to velocity fields of the form:

$$
\begin{gathered}
\tilde{u}_{n}=\frac{\left|A_{n}\right|}{\alpha} r^{\lambda_{n r}-1}\left\{\cos \left(\lambda_{n i} \log (r)+\chi_{n}\right) \tilde{g}_{n r}^{\prime}(\phi)-\sin \left(\lambda_{n i} \log (r)+\chi_{n}\right) \tilde{g}_{n i}^{\prime}(\phi)\right\}, \\
\tilde{v}_{n}=\left|A_{n} \lambda_{n}\right| r^{\lambda_{n r}-1}\left\{-\cos \left(\lambda_{n i} \log (r)+\chi_{n}+\beta_{n}\right) \tilde{g}_{n r}(\phi)\right. \\
\left.+\sin \left(\lambda_{n i} \log (r)+\chi_{n}+\beta_{n}\right) \tilde{g}_{n i}(\phi)\right\}
\end{gathered}
$$

where $A_{n}=\left|A_{n}\right| \mathrm{e}^{\mathrm{i} \chi_{n}}$ is a complex amplitude and $\beta_{n}=\arg \lambda_{n}$. Here we choose to index the modes such that $n=1,2,3, \ldots$ correspond to the modes with $\lambda_{(n+1) r}>\lambda_{n r}>0$ and $n=-1,-2,-3, \ldots$ correspond to $\lambda_{(n-1) r}<\lambda_{n r}<0$.

For $\alpha \ll 1$ and $R e-\operatorname{Re}_{c}=O(\alpha)$ the modes that prove of most interest are the complex conjugate pair for which $0<\lambda_{1 r}<2$ and the antisymmetric Drazin mode with $\lambda_{-2} \approx-1$. The first of these modes has a leading order velocity field in the form

$$
\begin{gathered}
\tilde{u}_{1}=\frac{\left|A_{1}\right|}{\alpha} r^{\lambda_{1 r^{-1}}} \cos \left(\lambda_{1 i} \log (r)+\chi_{1}\right) G^{\prime \prime}(\phi), \\
\tilde{v}_{1}=-\left|A_{1} \lambda_{1}\right| r^{\lambda_{1 r^{-1}}} \cos \left(\lambda_{1 i} \log (r)+\chi_{1}+\beta_{1}\right) G^{\prime}(\phi) .
\end{gathered}
$$

For a choice of $\chi_{1}=\pi / 2$ this radially oscillatory mode will satisfy the Dirichlet condition of $\tilde{u}=0$ at the outlet, $r=1$.

In figure 14 we show the radial dependence of the neutral finite-domain twodimensional eigenfunctions (at constant $\phi$ ) on the first four neutral curves for $\alpha=0.1$ and $\eta=100$. It is clear that, away from the inlet region, the bulk of the twodimensional finite-domain eigenfunction is in agreement with this radially oscillating Dean mode. The distinction between successive neutral curves is the number of half periods of the complex (radially oscillatory) Dean mode that can 'fit' into the chosen domain size $\eta^{-1}<r<1$. An additional bifurcation is obtained each time that $\lambda_{1 i}$ has increased enough for an extra half period of the (complex) similarity solution eigenmode to be accommodated in the finite domain. In the small $\alpha$ limit, $\lambda_{1 i}$ increases over the same short, $R e-R e_{c}=O(\alpha)$, range of Reynolds numbers and so the succession of neutral curves must cluster together as $\alpha \rightarrow 0$.

Simple geometric constraints mean that increasing the radius ratio leads to a decrease in the value of $\lambda_{1 i}$ required to fit a given number of half periods of the Dean mode into the domain. As $\eta$ increases, the critical Reynolds number of each bifurcation is observed to decrease accordingly; the limiting Reynolds number for $\eta \gg 1$ being that at which the complex conjugate eigenmode is formed. The finite-domain bifurcations must, therefore, always occur at Reynolds numbers distinct from (above) $R e_{c}$, but the separation is $O(\alpha)$ as $\alpha \rightarrow 0$. This single-mode explanation does not provide the complete picture because this one complex Dean mode cannot simultaneously satisfy the finite-domain boundary condition at the inlet, but nonetheless it is an excellent predictor of the bulk response.

To satisfy $\tilde{u}=\tilde{v}=0$ at the inlet we must also introduce a contribution from the (downstream relevant) Drazin mode,

$$
\tilde{u}_{-2}=\frac{A_{-2}}{\alpha} r^{\lambda_{-2}-1} G^{\prime \prime}(\phi) \quad \tilde{v}_{-2}=-\lambda_{-2} A_{-2} r^{\lambda_{-2}-1} G^{\prime}(\phi)
$$

at leading order. For this downstream decaying mode to cancel the influence of the complex conjugate mode at the inlet, leading to both $\tilde{u}$ and $\tilde{v}$ vanishing there, requires 


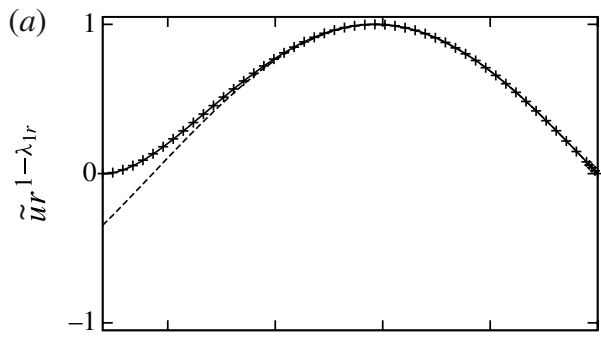

(b)
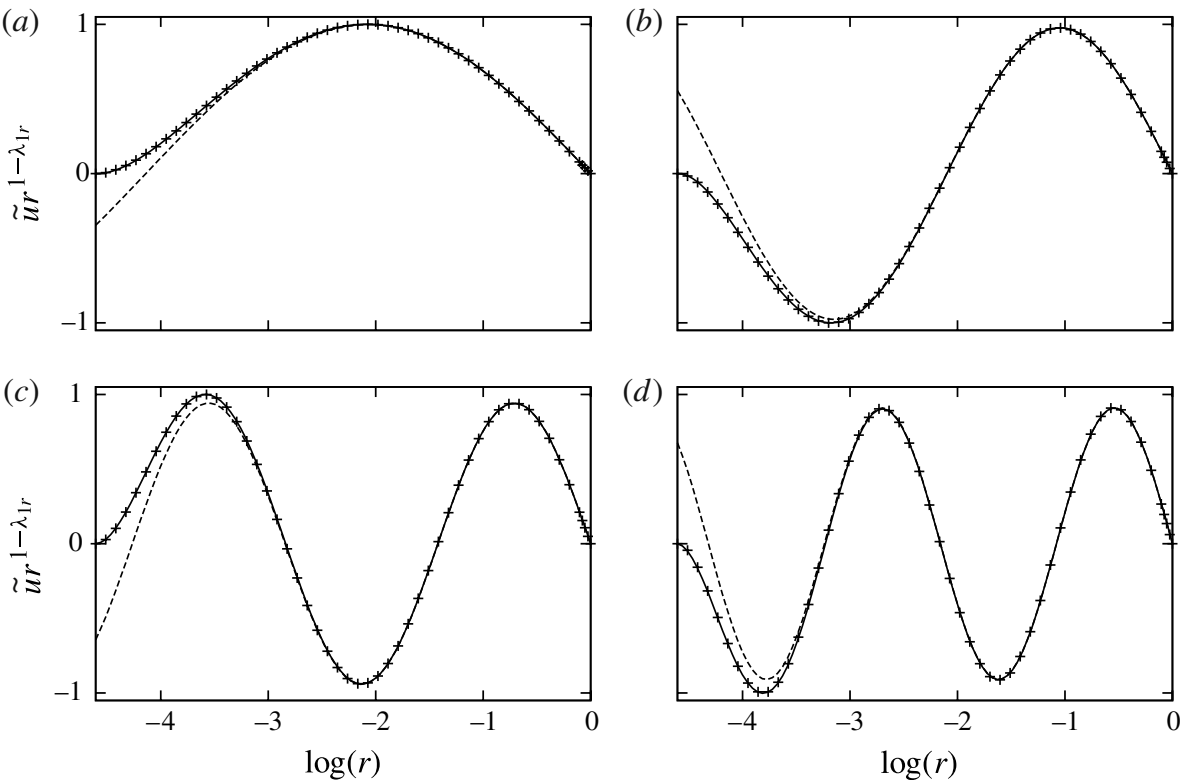

FIGURE 14. Radial slices (at $\phi=-0.5$ ) of the finite-domain critical eigenfunction for an exactly self similar base flow (data points). The critical eigenfunction, scaled by $r^{1-\lambda_{1 r}}$, is overlaid with predictions based upon a single Dean mode with complex eigenvalue $\lambda_{1}$ (dashed line) and the $\alpha \ll 1$ two-mode prediction that includes $\lambda_{-2}$ (solid line). Note that the twomode prediction is indistinguishable from the full numerical solution. The results shown are for $\alpha=0.1, \eta=100$ and the appropriate Jeffery-Hamel flow imposed as a Dirichlet condition at both the inlet and the outlet. (a) Neutral curve 1, $R e=94.27$ (b); neutral curve 2, $\operatorname{Re}=94.70 ;(c)$ neutral curve $3, \operatorname{Re}=95.45(d)$; neutral curve 4, $\operatorname{Re}=96.54$.

the following condition on its amplitude:

$$
\begin{array}{r}
\frac{\left|A_{1}\right|}{\alpha} \eta^{1-\lambda_{1 r}} \cos \left(-\lambda_{1 i} \log (\eta)+\frac{\pi}{2}\right)=-\frac{A_{-2}}{\alpha} \eta^{1-\lambda_{-2}}, \\
-\left|A_{1} \lambda_{1}\right| \eta^{1-\lambda_{1 r}} \cos \left(-\lambda_{1 i} \log (\eta)+\frac{\pi}{2}+\beta_{1}\right)=\lambda_{-2} A_{-2} \eta^{1-\lambda_{-2}},
\end{array}
$$

which reduces to

$$
\lambda_{-2} \sin \left(\lambda_{1 i} \log (\eta)\right)=\left|\lambda_{1}\right| \sin \left(\lambda_{1 i} \log (\eta)-\arg \lambda_{1}\right) .
$$

This condition determines the critical Reynolds number implicitly, since the values of the Dean exponents $\lambda_{1,-2}$ are functions of $R e$. At points where (6.10) is satisfied a temporally neutral two-dimensional eigenfunction may be constructed (to leading order in an expansion in terms of $\alpha$ ) from the two Dean modes discussed above. The eigenfunction obtained by combining these two modes is shown in figure 14 as a solid line. In each case, to the graphical accuracy shown, the prediction is indistinguishable from the finite-domain critical eigenfunction (the data points) over the entire domain.

A prediction for the location of finite-domain bifurcations can be obtained by solving the Dean eigenvalue problem and identifying Reynolds numbers for which $\lambda_{1}$ and $\lambda_{-2}$ satisfy (6.10). For $\alpha=0.1$ table 2 gives predictions, obtained from (6.10), for the first six critical Reynolds numbers together with the values determined from 

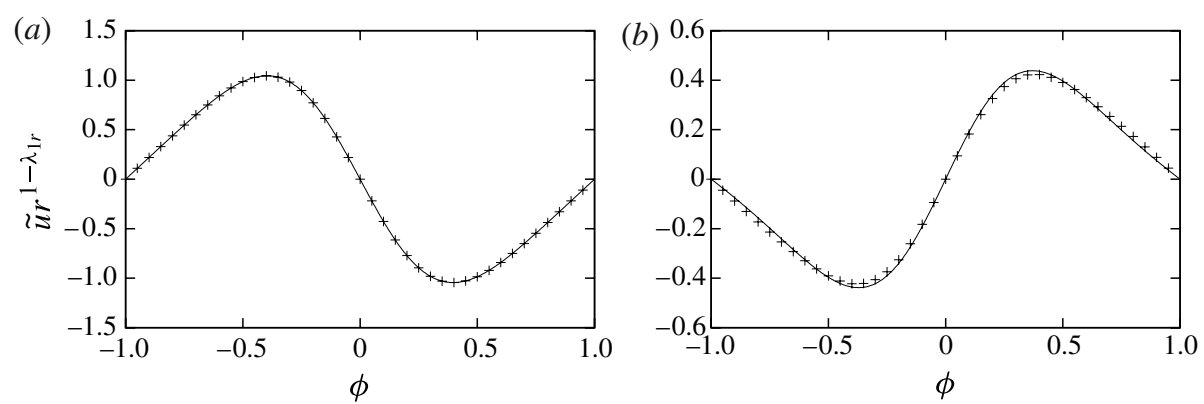

FIGURE 15. Azimuthal comparison (at $r \approx 0.1$ ) of the similarity solution eigenfunction (points) with the two Dean mode prediction (solid line). (a) Neutral curve 1, $R e=94.27$; (b) neutral curve $6, R e=99.82$. Results are otherwise for the same boundary conditions and parameter values as in figure 14.

$\begin{array}{lcccccc}\text { Neutral curve } & 1 & 2 & 3 & 4 & 5 & 6 \\ \text { Finite domain } \widehat{R e} & 94.27 & 94.70 & 95.45 & 96.54 & 97.99 & 99.82 \\ \text { Predicted value } & 94.27 & 94.70 & 95.45 & 96.55 & 98.02 & 99.87\end{array}$

TABLE 2. Critical Reynolds numbers for the first six finite-domain pitchfork bifurcations together with the predicted values at which (6.10) is satisfied. The critical Reynolds numbers are for the finite-domain problem with the similarity solution imposed as a Dirichlet condition at both the inlet and the outlet for $\alpha=0.1$ and $\eta=100$.

our numerical scheme. It is clear that the prediction for $\widehat{R e}_{c}$ at $\alpha=0.1$ becomes slightly less accurate for the higher modes, as indexed by the number of the neutral curve; however, for a fixed choice of neutral curve the agreement will be improved by considering lower values of $\alpha$. Figure 15 shows the azimuthal agreement between the finite-domain critical eigenfunction and the two mode prediction at $r \approx 0.1$ and $\alpha=0.1$. The agreement is significantly better for the first neutral curve than for the sixth at this value of wedge angle.

These observations hold only for a specific choice of boundary conditions that allow the base flow at bifurcation to be exactly Jeffery-Hamel (for the Dean modes to be obtained). However, the behaviour observed when the boundary conditions are perturbed remains qualitatively similar. For example, the effect of imposing a quadratic inlet condition is to create a base flow that is not quite self-similar in a small neighbourhood local to $r=\eta^{-1}$. In fact, this leads to less than a $1 \%$ difference in the observed critical Reynolds number when $\alpha=0.1$ and $\eta=100$ and the response in the bulk of the flow remains largely as described above.

\section{Discussion}

In this work we have described the solution structure for the flow in a wedgeshaped domain of finite radius ratio. The flow is governed by three parameters: the divergence angle $\alpha$, the radius ratio $\eta$ and the Reynolds number $R e$. A detailed examination of the numerical solutions at finite $\eta$ has revealed the critical values of $\alpha$ and $R e$ at which the symmetric outflow state becomes unstable to midplane symmetry-breaking global modes. Numerical continuation techniques have been used to determine additional critical boundaries at which the steady flow becomes unstable 
to temporally periodic modes (Hopf bifurcations) and at which solutions cease to exist (limit points). Figure 6 shows these critical points as functions of $\alpha$ and $R e$ at $\eta=100$, chosen to be a representative radius ratio for our results. Additional computations have been performed for $\eta \in[50,1000]$ with little qualitative changes observed to the general picture of figure 6 . We choose to present the details of $\eta=100$ because it is a finite, but still in some sense a 'large' domain, which can be compared with the infinite-domain solution and also realized in a laboratory setting.

The symmetric flow in a wedge of finite radial extent has been shown to become unstable at parameter values only a little beyond the boundary $\mathscr{B}_{2}$, predicted by the infinite-domain (Jeffery-Hamel) similarity solution. This result is in agreement with Sobey \& Drazin (1986) and Banks et al. (1988) who stated that the symmetric Jeffery-Hamel solution should not be used to predict the flow in a channel for $R e>R e_{c}$. The relatively good agreement on the location of the bifurcation between similarity solution and finite domain is in stark contrast to the failure of the (subcritical) similarity solution bifurcation to predict the criticality of the finite-domain bifurcation. Our results strongly reinforce the assertion of both Sobey \& Drazin (1986) and Dennis et al. (1997) that a supercritical pitchfork bifurcation represents the generic mechanism for the loss of stability of the symmetric flow in a two-dimensional smoothly expanding channel. These results are also in line with the literature for two-dimensional expansions in general (Cliffe \& Greenfield 1982; Fearn, Mullin \& Cliffe 1990). A supercritical pitchfork bifurcation has been found for a wide range of channel angles, and radius ratios and for a variety of distinct boundary conditions imposed at both the inlet and the outlet. The value of the critical Reynolds number for the finite-domain flow, $\widehat{R e}_{c}$, is found to be relatively insensitive to the precise choice of inlet/outlet boundary conditions and is particularly robust in the limit $\alpha \rightarrow 0$.

Thus, the similarity solution provides a good estimate of the critical Reynolds number for loss of midplane symmetry at small wedge angles, but fails to predict the criticality of this bifurcation and does not explain the existence of a sequence of bifurcations observed in the finite domain. In order to understand these unusual features of the problem, we have considered a closely related finite-domain problem where the inlet/outlet conditions are chosen such that the base flow is exactly selfsimilar, but for which the two-dimensional instability must vanish at the inlet and the outlet. By considering the spatial stability of the similarity solution we demonstrate that, for $\alpha \ll 1$, a global two-dimensional (temporal) eigenmode of the finite-domain flow can be constructed from the superposition of Dean eigenmodes of the infinitedomain problem. This construction is made particularly simple by the asymptotic properties of the Dean modes for $\alpha \ll 1$, namely the existence of multiple distinct modes with the same $\phi$ dependence; one of which is oscillatory in the radial coordinate. In the limit of $\alpha \ll 1$, complex (radially oscillatory) Dean modes occur within $O(\alpha)$ of the critical point $R e_{c}$. Therefore, the agreement between the critical Reynolds number predicted by the infinite-domain model and the corresponding finitedomain value is highly misleading; it is entirely a consequence of this asymptotic property. At bifurcation the radial behaviour of the symmetry-breaking eigenmode in a finite domain is a superposition of Dean modes rather than a single mode (as found in the infinite domain). The dominant contribution is from the same eigenmode as was responsible for the infinite-domain bifurcation. However, the variation at leading order of the associated eigenvalue over an $O(\alpha)$ range in the Reynolds number ensures that the radial behaviour of the eigenmode at the two bifurcation points is entirely distinct. Given this entirely different radial (oscillatory) behaviour, it is not surprising that the nonlinear interaction is different, leading to a supercritical bifurcation in the 
finite case. These same asymptotic scalings are also responsible for the presence of a sequence of discrete nested neutral curves for $\alpha \ll 1$ and $\eta \gg 1$. We have shown that the construction of global temporally unstable eigenmodes from the combination of two dominant (infinite-domain) Dean modes allows the accurate prediction of the finite-domain response.

As part of our investigation into the spatial stability of the similarity solutions we have provided numerical evidence for the conjecture first given by Banks et al. (1988) that the Dean modes provide two complete sets of eigenfunctions and that, when considering the evolution of a perturbation with respect to either increasing or decreasing radius, only one of these families is required. This interpretation of the Jeffery-Hamel spatial stability problem has been shown to accurately predict the behaviour of small perturbations imposed at either the inlet or the outlet of a finite domain of moderate radius ratio $(\eta=100)$.

Increasing the radius ratio results in a decrease in $\widehat{R e}_{c}$, the critical value for the first finite-domain bifurcation. This decrease is largest for values of $\alpha$ near to the 'nose' of neutral curve 1. For smaller values of $\alpha$ the effect is much less pronounced. However we note that, although $\operatorname{Re}_{c}$ and $\widehat{R e}_{c}$ appear to share the same limiting behaviour as $\alpha \rightarrow 0$, for a fixed value of $\alpha$ the finite-domain bifurcation is always at a Reynolds number slightly greater than $R e_{c}$. This difference in critical Reynolds numbers remains finite in the limit $\eta \rightarrow \infty$ when $\alpha=O(1)$.

For small channel angles and sufficiently large radius ratio $(\eta=100, \alpha<0.29)$ we have observed multiple stable flows in some Reynolds number ranges. We believe that this non-uniqueness of stable states explains the apparently contradictory observations that the evolution of the system is towards a flow on the symmetry-broken arm of a pitchfork bifurcation (Sobey \& Drazin 1986; Dennis et al. 1997) and a steady wave that is periodic on a logarithmic scale in $r$ (Tutty 1996; Kerswell et al. 2004). We observed a wave-like solution only for Reynolds numbers greater than a critical $(\alpha, \eta$ dependent) value associated with a limit point of isolated states. Our results strongly suggest that, for a small channel angle and sufficiently large radius ratio, these isolated states are finite-domain analogues of the solutions described by Kerswell et al. (2004); although in this case they do not arise by the same heteroclinic mechanism. These solutions are easy to obtain in the initial-value computations at sufficiently large Reynolds number, and we expect them to be visible in an experimental configuration of large enough radius ratio.

Although we have computed stable periodic orbits we have not been able to do so for the parameter values $(\alpha=0.1,0.3)$ cited by Hamadiche et al. (1994). We do not believe that stable periodic orbits exist at such small values of $\alpha$ but can offer no explanation for this discrepancy.

\section{Appendix. Dean modes in the limit $\alpha \ll 1$}

In the limit of small wedge angles the Jeffery-Hamel solution undergoes a subcritical symmetry-breaking bifurcation at the boundary $\mathscr{B}_{2}$. At this critical boundary, the base flow is

$$
G_{c}(\phi ; \alpha)=G_{c 0}(\phi)+\alpha^{2} G_{c 1}(\phi)+\cdots,
$$

and the $\mathscr{B}_{2}$ boundary is then defined by the critical Reynolds number

$$
\operatorname{Re}_{c}(\alpha)=\frac{3 \pi}{\alpha}+\alpha R_{c 1}+\cdots,
$$


where $R_{c 1} \approx-3.626$. If we now perturb about this critical boundary via

$$
R e=\operatorname{Re}_{c}(\alpha)+\alpha \bar{R},
$$

for some perturbation step $\bar{R}$, then the underlying Jeffery-Hamel base flow is described by the expansion

$$
G(\phi)=G_{c}(\phi ; \alpha)+\alpha^{2} \bar{G}(\phi)+\cdots,
$$

and spatial Dean eigenmodes, which satisfy (6.2), exist in the form

$$
\tilde{g}(\phi)=G_{c}^{\prime}(\phi ; \alpha)+\alpha^{2} \bar{g}(\phi)+\cdots
$$

with eigenvalues

$$
\lambda=\lambda_{0}+\alpha^{2} \lambda_{1}+\cdots
$$

The important point regarding the description (A 2) is that the eigenproblem for $\bar{g}$ determines the leading-order form of the eigenvalue, $\lambda_{0}$. The result of these scalings is that $\lambda_{0}=0,2$ when $\vec{R}=0$, however in general the eigenvalues vary at $O(1)$ whilst the eigenfunctions remain $G_{c}^{\prime}(\phi ; \alpha)$ to leading order. Full details of the numerical problem in this inner $R e-R e_{c}=O(\alpha)$ region can be found in Haines (2010).

In addition to the eigenmodes captured by the above expansion there are also the 'Drazin modes' (6.3) which have $\lambda=-1$ at $R e=R e_{c}$. In the limit of small wedge angle, $\alpha \ll 1$, the symmetry-breaking Drazin mode is also $\tilde{g}=G_{c}^{\prime}(\phi ; \alpha)$ to leading order. Unusually, this provides several discrete modes, with different radial dependence (via $\lambda$ ) but, to leading order, the same eigenfunction.

\section{REFERENCES}

AKulenko, L. D. \& Kumakshev, S. A. 2008 Bifurcation of multimode flows of a viscous fluid in a plane diverging channel. J. Appl. Math. Mech. 72 (3), 296-302.

BANKs, W. H. H., DraZin, P. G. \& ZATURSKA, M. B. 1988 On perturbations of Jeffery-Hamel flow. J. Fluid Mech. 186, 559-581.

Cliffe, K. A. \& GReEnfield, A. C. 1982 Some comments on laminar flow in symmetric two dimensional channels. Harwell Report AERE-TP 939.

Cliffe, K. A., Spence, A. \& TAVener, S. J. 2000 The numerical analysis of bifurcation problems with application to fluid mechanics. Acta Numerica 9, 39-131.

DeAn, W. R. 1934 Note on the divergent flow of fluid. Phil. Mag. 18 (7), 759-777.

Dennis, S. C. R., BAnKs, W. H. H., Drazin, P. G. \& ZATURSKA, M. B. 1997 Flow along a diverging channel. J. Fluid Mech. 336, 183-202.

DraziN, P. G. 1995 Stability of flow in a diverging channel. In Stability and Wave Propagation in Fluids and Solids (ed. G. P. Galdi). pp. 39-65. Springer.

Fearn, R. M., Mullin, T. \& Cliffe, K. A. 1990 Nonlinear flow phenomena in a symmetric sudden expansion. J. Fluid Mech. 211, 595-608.

FRAENKEL, L. E. 1962 Laminar flow in symmetrical channels with slightly curved walls. I. On the Jeffery-Hamel Solutions for flow between plane walls. Proc. R. Soc. Lond. Ser. A, Math. Phys. Sci. 267 (1328), 119-138.

Goldshtik, M., Hussain, F. \& Shtern, V. 1991 Symmetry breaking in vortex-source and Jeffery-Hamel flows. J. Fluid Mech. 232, 521-566.

Gresho, P. M. \& SANI, R. L. 1998 Incompressible Flow and the Finite Element Method: Volume 1, Advection-Diffusion and Isothermal Laminar Flow. John Wiley \& Sons.

HAines, P. E. 2010 The Jeffery-Hamel similarity solution and its relation to flow in a diverging channel. $\mathrm{PhD}$ thesis, The University of Manchester.

Hamadiche, M., Scott, J. \& JeAndel, D. 1994 Temporal stability of Jeffery-Hamel flow. $J$. Fluid Mech. 268, 71-88. 
HAMEL, G. 1916 Spiralförmige Bewegungen zäher Flüssigkeiten. Jahresbericht der Deutschen Mathematiker Vereinigung 25, 34-60.

Heil, M. \& Hazel, A. L. 2006 oomph-lib- An object-oriented multi-physics finite-element library. In Fluid-Structure Interaction (ed. M. Schafer \& H.-J. Bungartz). Lecture Notes in Computational Science and Engineering 53. pp. 19-49. Springer.

Hewitt, R. E. \& Hazel, A. L. 2007 Midplane-symmetry breaking in the flow between two counter-rotating disks. J. Engng Maths 57 (3), 273-288.

Jeffery, G. B. 1915 The two-dimensional steady motion of a viscous fluid. Phil. Mag. 6 (29), 455-465.

Kerswell, R. R., Tutty, O. R. \& DraZin, P. G. 2004 Steady nonlinear waves in diverging channel flow. J. Fluid Mech. 501, 231-250.

KuZnetsov, Y. A. 1998 Elements of Applied Bifurcation Theory. Springer.

PutKaradze, V. \& VorobiefF, P. 2006 Instabilities, bifurcations, and multiple solutions in expanding channel flows. Phys. Rev. Lett. 97 (14), 144502.

RosenheAD, L. 1940 The steady two-dimensional radial flow of viscous fluid between two inclined plane walls. Proc. R. Soc. Lond. Ser. A Math. Phys. Sci. (1934-1990) 175 (963), 436-467.

Salinger, A. G., Bou-Rabee, N. M., Pawlowski, R. P., Wilkes, E. D., Burroughs, E. A., LehoucQ, R. B. \& Romero, L. A. 2002 LOCA 1.0 Library of continuation algorithms: theory and implementation manual. Tech. Rep. SAND2002-0396. Sandia National Laboratories.

Sobey, I. J. \& Drazin, P. G. 1986 Bifurcations of two-dimensional channel flows. J. Fluid Mech. 171, 263-287.

Sobey, I. J. \& Mullin, T. 1993 Calculation of multiple solutions for the two-dimensional Navier-Stokes equations. Numer. Meth. Fluid Dyn. 4, 417.

TUtTy, O. R. 1996 Nonlinear development of flow in channels with non-parallel walls. J. Fluid Mech. 326, 265-284.

YU, Z., ShaO, X. \& LIN, J. 2010 Numerical computations of the flow in a finite diverging channel. J. Zhejiang Univ.-Science A 11 (1), 50-60.

ZIENKIEWICZ, O. C. \& ZHU, J. Z. 1992 The superconvergent patch recovery and a posteriori error estimates. Part 1: the recovery technique. Intl J. Numer. Meth. Engng 33 (7), 1331-1364. 